\title{
Single particle motion in colloidal dispersions: a simple model for active and nonlinear microrheology
}

\author{
By ADITYA S. KHAIR AND JOHN F. BRADY \\ Division of Chemistry and Chemical Engineering, California Institute of Technology, Pasadena, \\ CA 91125, USA
}

(Received 12 December 2004 and in revised form 9 December 2005)

The motion of a single Brownian probe particle subjected to a constant external body force and immersed in a dispersion of colloidal particles is studied with a view to providing a simple model for particle tracking microrheology experiments in the active and nonlinear regime. The non-equilibrium configuration of particles induced by the motion of the probe is calculated to first order in the volume fraction of colloidal particles over the entire range of $P e$, accounting for hydrodynamic and excluded volume interactions between the probe and dispersion particles. Here, $P e$ is the dimensionless external force on the probe, or Péclet number, and is a characteristic measure of the degree to which the equilibrium microstructure of the dispersion is distorted. For small $P e$, the microstructure (in a reference frame moving with the probe) is primarily dictated by Brownian diffusion and is approximately fore-aft symmetric about the direction of the external force. In the large $P e$ limit, advection is dominant except in a thin boundary layer in the compressive region of the flow where it is balanced by Brownian diffusion, leading to a highly non-equilibrium microstructure. The computed microstructure is employed to calculate the average translational velocity of the probe, from which the 'microviscosity' of the dispersion may be inferred via application of Stokes drag law. For small departures from equilibrium $(P e<1)$, the microviscosity 'force-thins' proportional to $P e^{2}$ from its Newtonian low-force plateau. For particles with long-range excluded volume interactions, force-thinning persists until a terminal Newtonian plateau is reached in the limit $P e \rightarrow \infty$. In the case of particles with very short-range excluded volume interactions, the force-thinning ceases at $P e \sim O(1)$, at which point the microviscosity attains a minimum value. Beyond $P e \sim O(1)$, the microstructural boundary layer coincides with the lubrication range of hydrodynamic interactions causing the microviscosity to enter a continuous 'force-thickening' regime. The qualitative picture of the microviscosity variation with $P e$ is in good agreement with theoretical and computational investigations on the 'macroviscosity' of sheared colloidal dispersions, and, after appropriate scaling, we are able to make a direct quantitative comparison. This suggests that active tracking microrheology is a valuable tool with which to explore the rich nonlinear rheology of complex fluids.

\section{Introduction}

Colloidal dispersions composed of micrometre-sized particles suspended in a viscous fluid are ubiquitous in everyday life: paints, emulsions, inks, slurries and foodstuffs being but a few examples. It is of particular importance to understand the mechanical 
response or flow behaviour of these materials induced by the application of external body forces and ambient flow fields. This is a difficult task as colloidal dispersions are typically viscoelastic or non-Newtonian in nature, i.e. they exhibit both viscous (liquidlike) and elastic (solid-like) traits depending on the length and time (or frequency) scales on which they are interrogated. The experimental and theoretical study of the flow behaviour of viscoelastic fluids, or rheology, has traditionally focused on the measurement of bulk properties such as shear viscosity, normal stress differences, and storage and loss moduli. Experiments are conducted in rheometers (e.g. coneand-plate, parallel-plate) where a macroscopic sample of the material is subjected to an oscillatory or steady shear flow. A review of traditional rheometry techniques may be found in Barnes, Hutton \& Walters (1989). There are several limitations to conventional rheometry: millilitre amounts of the substance under scrutiny are required; it is possible to sample only frequencies of the order of tens of Hertz (and hence the short-time dynamical response of the material cannot be probed); and the rheometer apparatus often suffers from mechanical inertia and slip at the walls.

The past decade or so has seen the emergence of a number of experimental procedures collectively known as 'microrheology', with the ability to measure viscoelastic properties of soft heterogeneous materials at the micrometre scale. Many diverse systems such as living cells, DNA, actin networks, gelatin and colloids near the glass transition have been investigated using microrheological techniques (for a review see Mackintosh \& Schimdt 1999; Waigh 2005). Microrheology does not suffer from several of the drawbacks that affect conventional 'macrorheology': microrheology requires only a small sample of the substance in comparison to macrorheology (a particular advantage in the case of rare biological materials); microrheology may be used to probe local viscoelastic properties (and hence serve to characterize inhomogeneous materials); and may sample frequencies up to the order of thousands of Hertz (and hence be used to study short-time dynamics of the material).

One of the most popular microrheology techniques involves the tracking of a single 'probe' particle to infer the properties of the embedding material. Typically, the probes are inert spherical beads of the order of a micrometre in radius. A passive tracking experiment may be performed where the change in probe location owing to random thermal fluctuations of the surrounding medium is monitored (with e.g. optical microscopy, light scattering, or laser-deflection particle tracking). The experimentally observed mean-squared displacement of the probe may be used to infer the complex shear modulus of the surrounding material via application of a frequency-dependent generalized Stokes-Einstein-Sutherland relation † (Mason \& Weitz 1995; Mason et al. 1997). Although this is a fairly standard experimental procedure, the validity of using the frequency-dependent generalized Stokes-Einstein-Sutherland relation has been called into question (Gittes et al. 1997). The major limitation of passive tracking experiments is that only linear viscoelastic properties may be ascertained. In contradistinction, active tracking experiments, in which the surrounding environment is driven out of equilibrium by application of an external force on the probe particle, may be used to study nonlinear viscoelastic properties of materials. (Note, our use of

$\dagger$ Recently, our attention has been brought to a little-known paper by W. Sutherland (1905), in which he derives the relationship between the translational diffusion coefficient and hydrodynamic mobility for an isolated spherical colloidal particle, or, as it is colloquially known, the 'StokesEinstein relation'. As Sutherland and Einstein published this fundamental result in the same year, 1905, we feel it only proper to acknowledge Sutherland's contribution; hence, we propose to call this the 'Stokes-Einstein-Sutherland relation'. 
the word active is not to be confused with active in the sense of biologically active suspensions containing self-propelled particles.) Motion of the probe particle may be induced by application of magnetic fields, optical tweezers, or by manufacturing the probe to be of a different density to its surrounding environment. Much less work has been conducted on active microrheology as compared to its passive counterpart; it is the aim of this study to construct a simple theoretical model for active microrheology, with a view to interpreting existing experimental results and guiding the design of new active tracking experiments. Indeed, an important question to address is to what extent can (or should) the results of an active tracking experiment be interpreted as a 'viscosity'.

As mentioned above, microrheology is able to probe the viscoelastic properties of materials that cannot be produced in sufficient quantity to allow macrorheological testing. This notwithstanding, it is important to determine to what degree (if at all) microrheological measurements are representative of the macroscopic, or bulk, properties of a material. Certainly, agreement between microrheologically and macrorheologically measured properties would lend support to the microrheological results; however, should agreement between micro and macro be expected? Furthermore, is such agreement necessary for microrheology to be useful? To answer these questions it must be appreciated that micro- and macro-rheology probe materials on different length scales: in microrheology the material is deformed on the scale of the probe (typically of the order of a micrometre), whereas in macrorheology the deformation is on a 'macroscopic' scale (e.g. the gap spacing, typically of the order of a millimetre or more, of a parallel-plate rheometer). (Note, in this discussion we are only considering single-particle, or 'one-point', microrheology; two-point microrheology (Crocker et al. 2000), which cross-correlates the fluctuating motion of two distant probes, may induce deformations on length scales much larger than the individual probe size.) Furthermore, in macrorheology the material is deformed using a viscometric flow field (e.g. simple shear), whilst in microrheology the flow induced by a moving probe is not viscometric. Thus, even in the large-probe (continuum) limit, micro and macro measurements may not agree. On a microstructural level there are also fundamental differences: macrorheology applies an ambient flow (or stress) field - a quadrupolar forcing -, whereas in microrheology a probe moves with a specified force (or velocity) - a dipolar forcing. With this in mind, we should not expect, in general, agreement between micro- and macro-rheological measurements, and great care must be taken in the interpretation of the microrheological results and comparison with macrorheological data. To this end, it is essential to develop accurate theoretical models for active microrheology experiments. A final point: discrepancies between micro- and macro-rheological data are indicative of the fundamental differences in the two techniques; by understanding such differences we can only learn more information about a particular material. Thus, microrheology should be viewed as a complement to, and not a replacement for, macrorheology.

Following the work of Squires \& Brady (2005), as a model for active microrheology we consider the motion of a single spherical probe particle under the imposition of a steady external force amidst a sea of force- and torque-free spherical colloidal bath particles. For simplicity, it is assumed that the probe particle is of the same size as the bath particles. The advective relative velocity field generated by application of the external force on the probe causes the spatio-temporal configuration or microstructure of the dispersion to be driven out of its equilibrium state. Counteracting this is the Brownian diffusion of particles caused by random thermal fluctuations of the solvent molecules, which acts to restore the equilibrium microstructure. The degree to which 
the microstructure is displaced from equilibrium is governed by the ratio of the magnitude of the external force to the Brownian force, known as the Péclet number, $P e$. The limit $P e \rightarrow 0$, in which the microstructure of the dispersion is primarily determined by Brownian diffusion, is the realm of passive (or linear) microrheology; when $P e$ is not small compared to unity, one is in the active (or nonlinear) regime.

The action of both advection and Brownian diffusion is strongly influenced by the hydrodynamic interactions between particles; thus, it is desirable to be able to investigate the effects of hydrodynamic interactions on the microstructure of the dispersion in a simple systematic fashion. To this end, an interparticle excluded volume interaction is introduced, by which particles are kept at a minimum separation of $2 b \geqslant 2 a$ apart, where $a$ is the true (or hydrodynamic) radius and $b$ the excluded volume (or thermodynamic) radius of an individual particle. The same 'excluded-annulus' model was used previously by Brady \& Morris (1997) and Bergenholtz, Brady \& Vicic (2002) in investigations on the microstructure and macrorheology of sheared suspensions. By altering the ratio $\hat{b}=b / a$, we are able to move continuously from the limits of no hydrodynamic interactions, $\hat{b} \rightarrow \infty$, to full hydrodynamic interactions, $\hat{b} \equiv 1$.

In order to make analytical progress it assumed that the dispersion is dilute (i.e. the volume fraction of background colloidal particles is small compared to unity) so that only interactions between the probe and a single background particle are important in establishing the microstructure. In this limit the pair-distribution function of the dispersion obeys a two-body Smoluchowski equation. Previous investigations on related problems have obtained only solutions to this Smoluchowski equation (for finite $\hat{b})$ in the limits of near equilibrium $(P e \ll 1)$ and non-colloidal $\left(P e^{-1} \equiv 0\right)$ dispersions. For small departures from equilibrium, Batchelor (1982), in a study of sedimentation in a dilute polydisperse suspension, determined the microstructure to first order in $P e$ for $\hat{b}=1$. Using this microstructure he calculates the average translational velocity of a particle in the dispersion, and in a subsequent paper (Batchelor 1983) exposes the relationship between the translational velocity and the self-diffusivity of a particle. At the other extreme $P e^{-1} \equiv 0$, Batchelor (1982) found that, for $\hat{b}=1$, the pair-distribution function is spherically symmetric about a reference particle. This is somewhat paradoxical given the directionality imposed by the external force (in Batchelor's case gravity) and the absence of Brownian diffusion, but is in fact a consequence of the fore-aft symmetry of the relative trajectories for a pair of particles in Stokes flow. In the absence of hydrodynamic interactions $(\hat{b} \rightarrow \infty)$, Squires \& Brady (2005) have derived an exact solution of the Smoluchowski equation for arbitrary Pe. In this study, the Smoluchowski equation is solved via a combination of perturbation methods and numerical computations, enabling us to determine the microstructure over the entire range of $P e$ and $\hat{b}$.

The pair-distribution function may be used to calculate quantities such as the microstructurally averaged translational velocity of the probe particle. The average velocity is an experimentally accessible quantity, as illustrated in the study of Habdas et al. (2004), who, using confocal microscopy, delineated the relationship between the average velocity and applied force for a magnetic particle moving in a dense colloidal dispersion. To facilitate a comparison with macrorheology experiments, we may interpret the average translational velocity of the probe particle in terms of a 'microviscosity' of the dispersion via application of Stokes drag law. In the case of non-colloidal suspensions, such a connection has been made by Davis \& Hill (1992) and Almog \& Brenner (1997) to the viscosity obtained from 'falling-ball' rheometry experiments. Theoretical calculations of the 'macroviscosity' of a sheared colloidal suspension have been reported by Bergenholtz et al. (2002) over the entire 
range of $P e$ (with $P e$ defined with the non-dimensional shear-rate in this case) and $\hat{b}$. In the limit $P e \rightarrow 0$ (regardless of the value of $\hat{b}$ ), they find the macroviscosity attains a low-shear Newtonian plateau, which, on increasing $P e$, is followed by a decrease, or 'shear-thinning', of the macroviscosity up to $P e \sim O(1)$. For $\hat{b}>1.1$, this shear-thinning persists on increasing $P e$ until a high-shear Newtonian plateau is reached in the limit $P e \rightarrow \infty$. However, for $\hat{b}<1.1$, the macroviscosity attains a minimum at $P e \sim O(1)$ and proceeds to grow, or 'shear-thicken', with increasing Pe. Squires \& Brady (2005) used their exact solution of the Smoluchowski equation in the absence of hydrodynamic interactions to compute the microviscosity of the dispersion for arbitrary Pe. They find that the microviscosity force-thins from a Newtonian plateau in the limit $P e \rightarrow 0$, until a second Newtonian plateau is reached as $P e \rightarrow \infty$, in qualitative agreement with the macroviscosity results of Bergenholtz et al. (2002). A major outcome of this work is the demonstration that the qualitative agreement between microviscosity and macroviscosity persists when the effects of hydrodynamic interactions between particles are included. Furthermore, after appropriate scaling, we are able to make a direct quantitative comparison between the micro- and macro-viscosity.

The remainder of this paper is set out as follows. In $\S 2$, we formulate the twobody Smoluchowski equation governing the spatio-temporal evolution of the nonequilibrium pair-distribution function. The separate hydrodynamic, Brownian and interparticle-force contributions to the ensemble-averaged translational velocity of the probe particle are derived in $\S 3$, along with the interpretation of the translational velocity as a microviscosity of the dispersion. Small departures from the equilibrium microstructure $(P e \ll 1)$ are the subject of $\S 4$. Here, in $\S 4.1$ we show that the distortion of the equilibrium microstructure may be calculated through terms of $O\left(P e^{2}\right)$ via a regular perturbation expansion, thereby extending the analysis of Batchelor (1982). To proceed to higher orders in $P e$ requires the use of matched asymptotic expansions. Section 4.2 is concerned with the linear-response (or passive) regime, where the perturbation to the equilibrium microstructure is linearly related to $\mathrm{Pe}$. In this limit, the microstructural evolution problem is identical to that for self-diffusion at long wavelengths (Russel, Saville \& Schowalter 1989; Brady 1994) and the microviscosity may be simply related to the long-time self-diffusivity of a particle. Moving to nonlinear response, in $\S 4.3$ we consider the effect of a nonlinear deformation to the microstructure on the microviscosity of the suspension. To obtain the non-equilibrium microstructure for arbitrary $P e$, we must solve the Smoluchowski equation numerically, as discussed in $\S 5$. The results of our numerical computations are presented in $\S 6$. To demonstrate the accuracy of the numerical solutions we focus first on the case of particles without hydrodynamic interactions, for which the Smoluchowski equation has been solved exactly (Squires \& Brady 2005). Next, we examine the effect of hydrodynamic interactions on the microstructure and microviscosity of the suspension. It is found that the degree of 'force-thickening' at large $P e$ may be tuned by altering $\hat{b}$, in agreement with the study of Bergenholtz et al. (2002) for the macroviscosity. Lastly, some concluding remarks are offered in $\S 7$.

\section{Non-equilibrium microstructure}

Consider an assemblage of $N$ spherical particles of radii $a$ homogeneously dispersed in an incompressible Newtonian suspending fluid of density $\rho$ and dynamic viscosity $\eta$. An external force, $\boldsymbol{F}^{\text {ext }}$, is applied to one of the particles (the probe) whilst the other $N-1$ background particles are force- and torque-free. An alternative procedure is to fix the velocity of the probe particle rather than the force imposed on it, as 
discussed by Squires \& Brady (2005) (see also Almog \& Brenner 1997). The fixedforce and fixed-velocity problems are different in detail, although they share similar qualitative features. The Reynolds number, $R e=\rho U a / \eta$ (with $U$ a typical velocity scale), characterizing the fluid inertia over a linear dimension of order of magnitude $a$, is assumed to be much less than unity, thus enabling use of the Stokes equations in describing the fluid flow. Our aim is to develop a theory that models the microstructure of the suspension. Specifically, it is desired to compute the pair-distribution function thus determining the probability of finding a background particle at a vector separation $\boldsymbol{r}$ from the probe.

Our point of departure is the Smoluchowski equation governing the spatiotemporal evolution of the probability distribution function $P_{N}\left(\boldsymbol{r}_{N}, t\right)$ of the $N$ particle configuration vector $\boldsymbol{r}_{N}$ :

$$
\frac{\partial P_{N}}{\partial t}+\sum_{i=1}^{N} \nabla_{i} \cdot j_{i}=0
$$

where the sum is over all particles in the suspension. The flux of particle $i$ is given by

$$
\boldsymbol{j}_{i}=\boldsymbol{U}_{i} P_{N}-\sum_{j=1}^{N} \boldsymbol{D}_{i j} P_{N} \cdot \nabla_{j}\left(\ln P_{N}+V_{N} / k T\right),
$$

where $\boldsymbol{U}_{i}$ is the hydrodynamic velocity due to the external force, $k T$ is the thermal energy, and $V_{N}$ is the $N$-particle interaction potential. The thermal or Brownian force acting on particle $i$ due to the random thermal fluctuations of the solvent molecules is $-k T \nabla_{i} \ln P_{N}$. The relative Brownian diffusivity of an $i j$-pair of particles is $\boldsymbol{D}_{i j}=k T \boldsymbol{M}_{i j}^{U F}$, where $\boldsymbol{M}_{i j}^{U F}$ is the hydrodynamic mobility tensor relating the velocity of particle $i$ to the force exerted on particle $j$.

At equilibrium, the absence of any external forcing implies that $\boldsymbol{U}_{i}=0$ for each particle, and the probability distribution (denoted as $P_{N}^{0}$ ) is independent of time. This results in a balance between the interparticle potential and thermal forces, $\ln P_{N}^{0}+V_{N} /$ $k T=0$, which is solved by the familiar Boltzmann distribution $P_{N}^{0} \sim \exp \left(-V_{N} / k T\right)$. Application of an external force to the probe particle will induce relative motion among the particles in the suspension, driving the system out of equilibrium and $P_{N}$ away from the Boltzmann distribution. The velocity of a particle $i$ due to such an externally imposed force on particle $j$ is given by $\boldsymbol{U}_{i}=\boldsymbol{M}_{i j}^{U F} \cdot \boldsymbol{F}_{j}^{e x t}$.

To arrive at a closed equation for the pair-distribution function, the $N$-particle Smoluchowski equation is integrated over the configurational degrees of freedom of $N-2$ particles, neglecting any resulting three-body interaction terms (for a detailed derivation see Squires \& Brady 2005). In discarding the three-body couplings, the validity of our theory is restricted to the limit of low background particle volume fraction, $\phi_{a}=4 \pi n a^{3} / 3 \ll 1$ (where $n$ is the number density of background particles), with the advantage that it is possible to make analytical progress. The pair-distribution function $g(\boldsymbol{r})$, defined as $n^{2} g(\boldsymbol{r})=((N-2) !)^{-1} \int P_{N}\left(\boldsymbol{r}_{N}, t\right) \mathrm{d} \boldsymbol{r}_{3} \ldots \mathrm{d} \boldsymbol{r}_{N}$, satisfies a pairlevel Smoluchowski equation:

$$
\frac{\partial g}{\partial t}+\nabla_{r} \cdot\left(\boldsymbol{U}_{r} g\right)=\nabla_{r} \cdot \boldsymbol{D}_{r} \cdot\left(g \nabla_{r} V / k T+\nabla_{r} g\right) .
$$

In writing (2.3), the centre-of-mass coordinate system of two particles $\boldsymbol{r}=\boldsymbol{r}_{2}-\boldsymbol{r}_{1}$ and $\boldsymbol{x}=\boldsymbol{r}_{2}+\boldsymbol{r}_{1}$ has been adopted, with $\boldsymbol{r}_{1}$ denoting the probe particle. The relative hydrodynamic velocity and relative Brownian diffusivity tensor are given by $\boldsymbol{U}_{r}=$ $\boldsymbol{U}_{2}-\boldsymbol{U}_{1}$ and $\boldsymbol{D}_{r}=\boldsymbol{D}_{11}+\boldsymbol{D}_{22}-\boldsymbol{D}_{12}-\boldsymbol{D}_{21}$, respectively. 


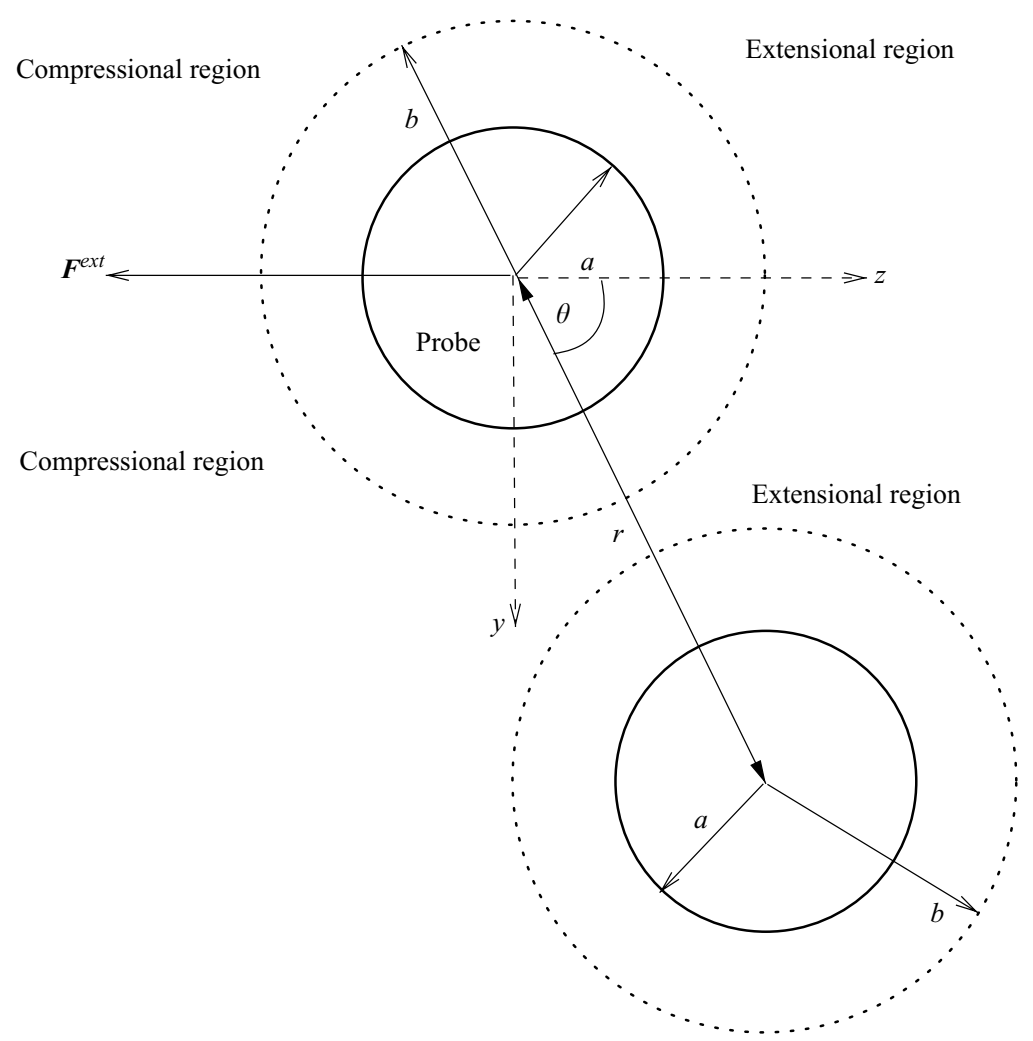

FIGURE 1. Definition sketch of the probe particle-background particle configuration.

The character of the pair-distribution function reflects the competition between the external forcing in driving the suspension out of equilibrium and Brownian diffusion which acts to restore equilibrium; both of these effects are heavily influenced by the nature of the hydrodynamic interactions between particles. Thus, in a theoretical model it is desirable to be able to tune the strength of the hydrodynamic interactions in a simple and systematic manner. To this end, the two-body interparticle potential $V(r)$ is chosen to be a simple 'excluded-annulus' model:

$$
V(r)= \begin{cases}\infty & \text { if } r \leqslant 2 b \\ 0 & \text { if } r>2 b\end{cases}
$$

The length $b(\geqslant a)$ is the excluded, or 'thermodynamic', radius of a particle, so that the separation between the probe particle and a background particle may be no less than $2 b$. Interactions of this nature may arise from e.g. surface asperities, grafted polymer chains, or electrostatic forces. The excluded-annulus model has been employed by Brady \& Morris (1997) and Bergenholtz et al. (2002) in computing the microstructure of a sheared suspension. A definition sketch of the two-sphere configuration is provided in figure 1 . Altering the parameter $\hat{b}=b / a \in[1, \infty)$ allows us to examine the role of hydrodynamic interactions in setting the microstructure. In the limit $\hat{b} \rightarrow \infty$, the particles do not experience hydrodynamic interactions and we recover the special case of a thermodynamic hard-sphere suspension; when $\hat{b} \equiv 1$, the particles experience full hydrodynamic interactions with one another. The diluteness 
assumption now requires the volume fraction based on the excluded radius $b$ to be small, $\phi_{b}=4 \pi n b^{3} / 3 \ll 1$.

The pair-level Smoluchowski equation is made dimensionless by scaling quantities as

$$
\boldsymbol{r} \sim b, \quad \boldsymbol{U} \sim \frac{F_{0}}{6 \pi \eta a}, \quad \boldsymbol{D} \sim 2 D, \quad t \sim \frac{6 \pi \eta a b}{F_{0}},
$$

where $F_{0}$ is the magnitude of the external force $\boldsymbol{F}^{e x t}$ and $D=k T / 6 \pi \eta a$ is the StokesEinstein-Sutherland diffusivity of an isolated colloidal particle of radius $a$. In this study, we consider time-independent microstructures for which the scaled pair-level Smoluchowski equation reads

$$
P e_{b} \nabla \cdot(\boldsymbol{U} g)=\nabla \cdot \boldsymbol{D} \cdot \nabla g,
$$

where all quantities are dimensionless, and for brevity the subscripts on $\nabla_{r}, \boldsymbol{U}_{r}$ and $\boldsymbol{D}_{r}$ have been dropped. The above equation reflects the competition between advection due to the application of an external force on the probe particle (the left-hand side of (2.6)) in driving the system out of equilibrium and Brownian motion (the right-hand side of (2.6)) in attempting to restore equilibrium. The degree to which the microstructure is distorted from its equilibrium state is governed by the Péclet number, $P e_{b}=F_{0} /(2 k T / b)$, which emerges naturally from the scaling. The subscript $b$ indicates that the Péclet number is based on the excluded radius $b$ rather than the hydrodynamic radius $a$. The Péclet number may be viewed as a ratio of forces: the external force $F_{0}$ over the Brownian force $2 k T / b$, or alternatively, as a ratio of time scales: the diffusive time $\tau_{D}=b^{2} / 2 D$ divided by the advective time $\tau_{A}=6 \pi \eta a b / F_{0}$. Either way, it should be clear that increasing the Péclet number corresponds to driving the system away from equilibrium.

To fully determine the pair-distribution function, the Smoluchowski equation (2.6) must be accompanied by appropriate boundary conditions. It is assumed that the suspension lacks any long-range order, which implies that

$$
g(s) \rightarrow 1 \text { as } s \rightarrow \infty,
$$

where $s=r / b$. The effect of the interparticle potential requires that the radial component of the relative flux is zero at $r=2 b$; thus, we have

$$
\hat{\boldsymbol{s}} \cdot \boldsymbol{D} \cdot \nabla g=P e_{b} \hat{\boldsymbol{s}} \cdot \boldsymbol{U} g \quad \text { at } \quad s=2,
$$

with $\hat{\boldsymbol{s}}=s / s$ the radial unit vector. As the pair-distribution function approaches unity at large distances it is useful to define the structural deformation function $f(s) \equiv g(s)-1$. Furthermore, in the dilute limit as the equilibrium pair-distribution function is unity everywhere (i.e. for $s \geqslant 2$ ), the structural deformation function is the departure from equilibrium caused by application of the external force on the probe.

\section{Average velocity of the probe particle and its interpretation as a microviscosity}

At low Reynolds number the average velocity of the probe particle may be written as

$$
\langle\boldsymbol{U}\rangle=\boldsymbol{U}^{0}+\left\langle\boldsymbol{U}^{H}\right\rangle+\left\langle\boldsymbol{U}^{P}\right\rangle+\left\langle\overline{\boldsymbol{U}^{B}}\right\rangle,
$$

where $\boldsymbol{U}^{0}=\boldsymbol{F}^{\text {ext }} / 6 \pi \eta a$ is the velocity of the probe particle in isolation. The presence of background colloidal particles causes the average velocity of the probe to differ 
from $\boldsymbol{U}^{0}$. This difference may be expressed as the sum of hydrodynamic $\left\langle\boldsymbol{U}^{H}\right\rangle$, interparticle $\left\langle\boldsymbol{U}^{P}\right\rangle$, and Brownian $\left\langle\overline{\boldsymbol{U}^{B}}\right\rangle$ contributions. In (3.1), the angle brackets denote an ensemble average over the admissible positions of a background particle, and the overbar on $\left\langle\overline{\boldsymbol{U}^{B}}\right\rangle$ denotes an average over the many collisions of the probe and background particles with the surrounding solvent molecules. In this section, we derive expressions for each of the three contributions.

The velocity of particle 1 ( $\boldsymbol{U}_{1}$ say) subjected to an external force $\boldsymbol{F}_{1}$ in the presence of particle 2 subject to another external force $\boldsymbol{F}_{2}$ is

$$
\boldsymbol{U}_{1}=\boldsymbol{M}_{11}^{U F} \cdot \boldsymbol{F}_{1}+\boldsymbol{M}_{12}^{U F} \cdot \boldsymbol{F}_{2} .
$$

In the present case where the particles are spherical and of equal size, the mobility tensors take the form

$$
\boldsymbol{M}_{i j}^{U F}=\frac{1}{6 \pi \eta a}\left\{A_{i j}(\hat{b} s) \hat{\boldsymbol{s}} \hat{\boldsymbol{s}}+B_{i j}(\hat{b} s)(\boldsymbol{I}-\hat{\boldsymbol{s}} \hat{\boldsymbol{s}})\right\},
$$

where $\boldsymbol{I}$ is the identity tensor, and $A_{i j}(r)$ and $B_{i j}(r)$ are scalar mobility functions that depend only on the magnitude of the dimensionless separation between the particles. Following the notation of Batchelor (1982), the relative Brownian diffusivity tensor and relative velocity are given by

$$
\begin{aligned}
\boldsymbol{D} & =G(\hat{b} s) \hat{\boldsymbol{s}} \hat{\boldsymbol{s}}+H(\hat{b} s)(\boldsymbol{I}-\hat{\boldsymbol{s}} \hat{\boldsymbol{s}}), \\
\boldsymbol{U} & =\{G(\hat{b} s) \hat{\boldsymbol{s}} \hat{\boldsymbol{s}}+H(\hat{b} s)(\boldsymbol{I}-\hat{\boldsymbol{s}} \hat{\boldsymbol{s}})\} \cdot\left(-\hat{\boldsymbol{F}}^{e x t}\right),
\end{aligned}
$$

where $\hat{\boldsymbol{F}}^{\text {ext }}=\boldsymbol{F}^{\text {ext }} / F_{0}$. The absence of a factor of 2 multiplying the right-hand side of $(3.4 a)$ is due to the relative diffusivity tensor being scaled with $2 D$ (the relative diffusivity of a pair of isolated spheres) rather than $D$ (the Stokes-Einstein-Sutherland diffusivity of a single isolated sphere). The hydrodynamic functions $G(r)$ and $H(r)$ describe the relative mobility parallel and transverse to the line of centres of a pair of spheres, respectively, and are defined by

$$
\begin{gathered}
G(r)=A_{11}(r)-A_{12}(r), \\
H(r)=B_{11}(r)-B_{12}(r) .
\end{gathered}
$$

The velocity of the probe particle caused by the application of the external force is $\boldsymbol{M}_{11}^{U F} \cdot \boldsymbol{F}^{\text {ext }}$; hence, the velocity due to hydrodynamic interactions is simply

$$
\boldsymbol{U}^{H}=\frac{\boldsymbol{F}^{e x t}}{6 \pi \eta a} \cdot\left\{A_{11}(\hat{b} s) \hat{\boldsymbol{s}} \hat{\boldsymbol{s}}+B_{11}(\hat{b} s)(\boldsymbol{I}-\hat{\boldsymbol{s}} \hat{\boldsymbol{s}})-\boldsymbol{I}\right\}
$$

i.e. the difference between the total velocity $\boldsymbol{M}_{11}^{U F} \cdot \boldsymbol{F}^{\text {ext }}$ and the velocity in isolation $\boldsymbol{U}^{0}$. To obtain the average velocity due to hydrodynamic interactions, the configurationspecific velocity $\boldsymbol{U}^{H}$ is weighted by the probability that the probe particle and a background particle are in a configuration characterized by the vector separation $s$ (namely $n g(s)$ ) and averaged over the ensemble of all possible configurations. Following this program we have

$$
\left\langle\boldsymbol{U}^{H}\right\rangle=\frac{3 \phi_{b}}{4 \pi} \frac{\boldsymbol{F}^{e x t}}{6 \pi \eta a} \cdot \int_{s \geqslant 2}\left\{A_{11}(\hat{b} s) \hat{\boldsymbol{s}} \hat{\boldsymbol{s}}+B_{11}(\hat{b} s)(\boldsymbol{I}-\hat{\boldsymbol{s}} \hat{\boldsymbol{s}})-\boldsymbol{I}\right\} g(\boldsymbol{s}) \mathrm{d} \boldsymbol{s} .
$$

It is important to note for large $s$ that $U^{H} \sim O\left(s^{-4}\right)$ and $g(s) \sim O(1)$; thus, the integral in (3.7) is convergent.

Suppose that the probe particle experiences an interparticle-force interaction with a background particle specified by the interparticle force $\boldsymbol{F}^{P}$; the average velocity of 
the probe due to this interparticle force is given by

$$
\left\langle\boldsymbol{U}^{P}\right\rangle=\frac{1}{6 \pi \eta a} \frac{3 \phi_{b}}{4 \pi} \int_{s \geqslant 2}\{G(\hat{b} s) \hat{\boldsymbol{s}} \hat{\boldsymbol{s}}+H(\hat{b} s)(\boldsymbol{I}-\hat{\boldsymbol{s}} \hat{\boldsymbol{s}})\} \cdot \boldsymbol{F}^{P}(\boldsymbol{s}) g(\boldsymbol{s}) \mathrm{d} \boldsymbol{s} .
$$

The excluded-annulus model is represented by a hard-sphere force $\boldsymbol{F}^{P}=-(k T / 2 b)$ $\delta(s-2) \hat{\boldsymbol{s}}$, where $\delta(x)$ is the Dirac delta distribution. Substituting this into (3.8) we have

$$
\left\langle\boldsymbol{U}^{P}\right\rangle=-\frac{F_{0}}{6 \pi \eta a} \frac{3 \phi_{b}}{4 \pi} \frac{2 G(2 \hat{b})}{P e_{b}} \oint_{s=2} g(s) \hat{\boldsymbol{s}} \mathrm{d} \Omega .
$$

An immediate consequence of (3.9) is that in the limit $\hat{b} \rightarrow 1$, where $G(2 \hat{b}) \sim \hat{b}-1$, $\left\langle\boldsymbol{U}^{P}\right\rangle \rightarrow 0$. This is a statement of the fact that the hard-sphere force plays no dynamical role in the case $\hat{b} \equiv 1$ : the rigidity of the particles is realized by the vanishing relative radial mobility.

Lastly, we consider the average velocity contribution of the probe particle due to Brownian motion. In Appendix $\mathrm{A}$ it is shown that

$$
\overline{U^{B}}=-\frac{1}{2} \nabla \cdot \boldsymbol{D},
$$

where the divergence is taken with respect to the last index of the relative diffusivity tensor. Averaging (3.10) over the ensemble of admissible two-particle configurations yields

$$
\left\langle\overline{\boldsymbol{U}^{B}}\right\rangle=-\frac{F_{0}}{6 \pi \eta a} \frac{3 \phi_{b}}{4 \pi} \frac{1}{P e_{b}} \int_{s \geqslant 2}\left(\frac{G(\hat{b} s)-H(\hat{b} s)}{s}+\frac{1}{2} \frac{\mathrm{d} G(\hat{b} s)}{\mathrm{d} s}\right) g(s) \hat{\boldsymbol{s}} \mathrm{d} s .
$$

The same result may be derived if we suppose the effect of Brownian motion is equivalent to the action of equal and opposite 'thermodynamic forces' $\boldsymbol{F}_{1}^{B}=k T \nabla \ln g(\boldsymbol{s})$ and $\boldsymbol{F}_{2}^{B}=-\boldsymbol{F}_{1}^{B}$ acting on the probe and a background particle, respectively (Batchelor 1982). Note, the integrand in (3.11) is of $O\left(s^{-5}\right)$ for large $s$; hence, the integral is convergent.

Aside from the external force there are no other directional influences on $g(s)$; therefore, $g(s)$ is axially symmetric about the orientation of $\boldsymbol{F}^{\text {ext }}$. Moreover, as $\boldsymbol{U}^{0}$ is parallel to $\boldsymbol{F}^{\text {ext }}$, we expect $\left\langle\boldsymbol{U}^{H}\right\rangle,\left\langle\boldsymbol{U}^{P}\right\rangle$, and $\left\langle\overline{\boldsymbol{U}^{B}}\right\rangle$ to be parallel to $\boldsymbol{F}^{\text {ext }}$ also. With this in mind, we are able to interpret the change in translational velocity of the probe owing to the presence of the background particles as a dimensionless relative microviscosity, $\eta_{r}$, of the suspension. This is done by application of the Stokes drag formula $\boldsymbol{F}^{e x t} / 6 \pi \eta a=\eta_{r}\langle\boldsymbol{U}\rangle$. Thus, the relative microviscosity is defined by

$$
\eta_{r} \equiv \frac{F_{0}}{6 \pi \eta a \hat{\boldsymbol{F}}^{e x t} \cdot\langle\boldsymbol{U}\rangle} .
$$

Note, the microviscosity contains (through its dependence on $\langle\boldsymbol{U}\rangle$ ) an a priori unknown dependence on the probe-to-background particle size ratio; this fact must be appreciated when analysing results from an active tracking experiment. In our study the probe and background particles are of equal size so this is not a concern (see, however, the discussion in $\S 7$ and Squires \& Brady 2005).

For dilute dispersions the denominator in (3.12) can be expanded to first order in the background particle volume fraction $\phi_{b}$, and the relative microviscosity may be written as $\eta_{r}=1+\eta_{i} \phi_{b}$, where $\eta_{i}=\eta_{i}^{H}+\eta_{i}^{P}+\eta_{i}^{B}$ is the intrinsic microviscosity (i.e. the relative microviscosity minus the Newtonian solvent contribution and normalized by the background particle volume fraction); $\eta_{i}^{H}, \eta_{i}^{P}$ and $\eta_{i}^{B}$ are the hydrodynamic, 
interparticle and Brownian contributions to the intrinsic microviscosity, respectively. A question we shall explore later is the relation between this microviscosity and the macroviscosity determined from studies on macroscopically sheared colloidal dispersions.

To highlight the role played by the non-equilibrium microstructure it is instructive to express the intrinsic microviscosity contributions in terms of the structural deformation function $f(s)$. First, for the intrinsic hydrodynamic microviscosity we have

$$
\eta_{i}^{H}=\eta_{i, 0}^{H}-\frac{3}{4 \pi} \hat{\boldsymbol{F}}^{\text {ext }} \hat{\boldsymbol{F}}^{\text {ext }}: \int_{s \geqslant 2}\left\{A_{11}(\hat{b} s) \hat{\boldsymbol{s}} \hat{\boldsymbol{s}}+B_{11}(\hat{b} s)(\boldsymbol{I}-\hat{\boldsymbol{s}} \hat{\boldsymbol{s}})-\boldsymbol{I}\right\} f(\boldsymbol{s}) \mathrm{d} \boldsymbol{s},
$$

where $\eta_{i, 0}^{H}$ is the contribution to the intrinsic hydrodynamic microviscosity due to the equilibrium microstructure:

$$
\eta_{i, 0}^{H}=-\int_{2}^{\infty}\left(A_{11}(\hat{b} s)+2 B_{11}(\hat{b} s)-3\right) s^{2} \mathrm{~d} s .
$$

The intrinsic interparticle microviscosity takes the form

$$
\eta_{i}^{P}=\frac{3}{4 \pi} \frac{2 G(\hat{b} s)}{P e_{b}} \hat{\boldsymbol{F}}^{e x t} \cdot \oint_{s=2} f(s) \hat{\boldsymbol{s}} \mathrm{d} \Omega
$$

from which we see the equilibrium microstructure does not affect $\eta_{i}^{P}$. For the intrinsic Brownian microviscosity we have

$$
\eta_{i}^{B}=\frac{3}{4 \pi} \frac{1}{P e_{b}} \hat{\boldsymbol{F}}^{e x t} \cdot \int_{s \geqslant 2}\left(\frac{G(\hat{b} s)-H(\hat{b} s)}{s}+\frac{1}{2} \frac{\mathrm{d} G(\hat{b} s)}{\mathrm{d} s}\right) f(s) \hat{\boldsymbol{s}} \mathrm{d} \boldsymbol{s},
$$

which, as in the case of the intrinsic interparticle microviscosity, depends solely on the non-equilibrium microstructure.

\section{Non-equilibrium microstructure and microrheology at small $\boldsymbol{P e}_{b}$}

\subsection{Perturbation expansion of the structural deformation}

At small Péclet number, when the ratio of the external force to the restoring Brownian force is much less than unity, the suspension is only slightly displaced from its equilibrium state, enabling the pair-distribution function to be calculated via a perturbation expansion in $P e_{b}$. Recalling the definition of $P e_{b}$ as a ratio of time scales, we anticipate that the perturbation to the equilibrium microstructure is singular, based on the general non-uniformity criterion proposed by Van Dyke (1975, pp. 80-83). Before dealing with the complicating effect of hydrodynamic interactions, it is useful to examine the singular nature of the problem in their absence. Neglecting hydrodynamic interactions, the pair-level Smoluchowski equation (2.6) and associated boundary conditions $(2.7)$ and $(2.8)$ reduce to

$$
\begin{gathered}
P e_{b} \hat{\boldsymbol{U}} \cdot \nabla f=\nabla^{2} f, \\
P e_{b}(\hat{\boldsymbol{s}} \cdot \hat{\boldsymbol{U}})(1+f)=\frac{\mathrm{d} f}{\mathrm{~d} s} \quad \text { at } \quad s=2, \\
f \rightarrow 0 \text { as } s \rightarrow \infty,
\end{gathered}
$$

where $\hat{\boldsymbol{U}}=-\hat{\boldsymbol{F}}^{\text {ext }}$. The distortion to the equilibrium microstructure is governed by a balance between isotropic diffusion and advection by a constant relative 'velocity' $\hat{\boldsymbol{U}}$. Even though the Péclet number is small, from $(4.1 a)$ we see that at distances $s \sim O\left(P e_{b}^{-1}\right)$ (where hydrodynamic interactions are unimportant anyway) the effects 
of advection and diffusion are of the same order of magnitude. Defining an 'outer' coordinate $\rho=s P e_{b} \sim O(1)$ we see that (4.1a) takes the form

$$
\hat{\boldsymbol{U}} \cdot \nabla_{\rho} F=\nabla_{\rho}^{2} F,
$$

in this outer region; for clarity, we denote the structural deformation in the outer region as $F$. It is required that a solution to (4.2) must vanish as $\rho \rightarrow \infty$ and match with the solution of the 'inner' equation (4.1a) as $\rho \rightarrow 0$. If $P e_{b} \equiv 0$, then the uniformly valid solution is simply $f=0$, corresponding to an equilibrium microstructure.

In the inner region, the first perturbation to the equilibrium microstructure is linear in the forcing $\hat{U}$ and is given by

$$
f=-P e_{b} \frac{4}{s^{2}} \hat{\boldsymbol{s}} \cdot \hat{\boldsymbol{U}}
$$

which has the character of a diffusive dipole directed along $\hat{\boldsymbol{F}}^{\text {ext }}$. In terms of the outer variables, (4.3) is

$$
f=-P e_{b}^{3} \frac{4}{\rho^{2}} \hat{\boldsymbol{\rho}} \cdot \hat{\boldsymbol{U}}
$$

thus, the leading-order perturbation to the equilibrium microstructure is of $O\left(P e_{b}^{3}\right)$ in the outer region. This indicates that the expansion in the inner region will be regular through terms of $O\left(P e_{b}^{2}\right)$; specifically, we may write

$$
f=f_{1} P e_{b}+f_{2} P e_{b}^{2}+O\left(P e_{b}^{3}\right),
$$

where $f_{1}$ is given by (4.3). Now, $f_{2}$ must be quadratic in the forcing $\hat{\boldsymbol{U}}$ and it is a simple (if tedious) matter to show that

$$
f_{2}=2\left(\frac{4}{s^{3}}-\frac{1}{s}\right) \hat{\boldsymbol{s}} \hat{\boldsymbol{s}}: \hat{\boldsymbol{U}} \hat{\boldsymbol{U}}-2\left(\frac{4}{3 s^{3}}-\frac{1}{s}\right),
$$

from which it is seen that, to leading order, $f_{2} P e_{b}^{2} \sim O\left(P e_{b}^{3}\right)$ in the outer region also; hence, the first term in the outer expansion should be matched to $f_{1} P e_{b}+f_{2} P e_{b}^{2}$. Now, if we suppose the next term in the inner expansion to be $f_{3} P e_{b}^{3}$, then the singular nature of the expansion is revealed, because the particular solution of $f_{3}$ is forced by gradients in $f_{2}$, which from (4.6) are to leading order $O\left(s^{-2}\right)$. The resulting particular solution for $f_{3}$ does not decay as $s \rightarrow \infty$. The matching condition for $f_{3}$ is: $f_{1} P e_{b}+f_{2} P e_{b}^{2}+f_{3} P e_{b}^{3}=F_{1} P e_{b}^{3}$ in the limits $\rho \rightarrow 0$ and $s \rightarrow \infty$, where $F_{1}$ is the first term in the outer solution. Although we may continue to higher orders in the expansion, the system (4.1a)-(4.1c) can be solved exactly (Squires \& Brady 2005), making this unnecessary.

We now consider the effect of hydrodynamic interactions. Guided by the analysis above we propose an expansion for the structural deformation in the inner region of the form

$$
f=P e_{b} \hat{\boldsymbol{s}} \cdot \hat{\boldsymbol{F}}^{\text {ext }} f_{1}(s)+P e_{b}^{2}\left(\hat{\boldsymbol{s}} \hat{\boldsymbol{s}}: \hat{\boldsymbol{F}}^{\text {ext }} \hat{\boldsymbol{F}}^{\text {ext }} f_{2}(s)+h_{2}(s)\right)+O\left(P e_{b}^{3}\right) .
$$

Substituting this expansion into the Smoluchowski equation (2.6) and boundary conditions (2.7) and (2.8), we obtain at $O\left(P e_{b}\right)$ the system

$$
\begin{gathered}
\frac{\mathrm{d}}{\mathrm{d} s}\left(s^{2} G(\hat{b} s) \frac{\mathrm{d} f_{1}}{\mathrm{~d} s}\right)-2 H(\hat{b} s) f_{1}=-s^{2} W(\hat{b} s), \\
\frac{\mathrm{d} f_{1}}{\mathrm{~d} s}=-1 \text { at } s=2, \\
f_{1} \rightarrow 0 \text { as } s \rightarrow \infty,
\end{gathered}
$$




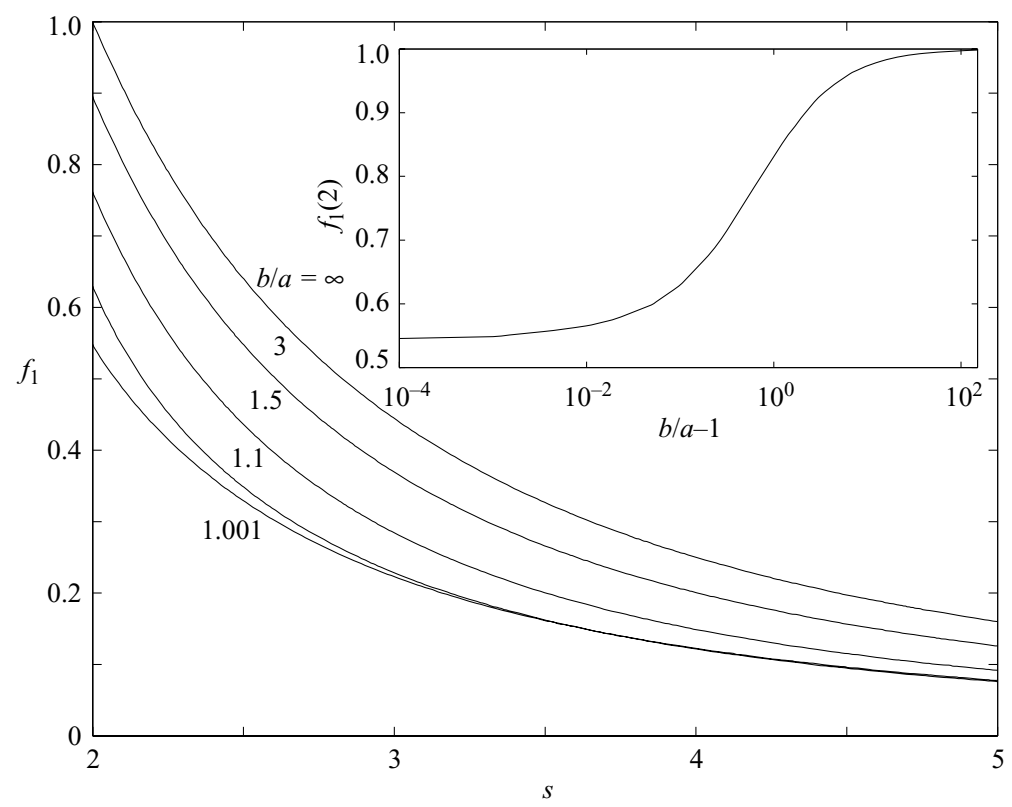

Figure 2. The $O\left(P e_{b}\right)$ structural deformation function $f_{1}$ for several values of $\hat{b}=b / a$. The inset plots the contact value of $f_{1}$, i.e. $f_{1}(2)$, versus $\hat{b}$. In the limit $\hat{b} \rightarrow 1$ we find $f_{1}(2)=0.545$, in good agreement with Batchelor \& Wen (1982) (see figure 9 in their paper).

where $W(r)=\mathrm{d} G / \mathrm{d} r+2(G-H) / r$, is proportional to the divergence of the relative velocity. To solve this equation we note that in the far field

$$
f_{1}=\frac{f_{1}^{\infty}}{s^{2}}+\frac{3}{8 \hat{b}}\left(3 f_{1}^{\infty}-\frac{5}{\hat{b}^{4}}\right) \frac{1}{s^{3}}+\frac{9}{20 \hat{b}^{2}}\left(3 f_{1}^{\infty}-\frac{5}{\hat{b}^{4}}\right) \frac{1}{s^{4}}+O\left(s^{-5}\right),
$$

reflecting the dipole nature of the disturbance. Using (4.9) as the 'initial condition', (4.8a) is integrated backwards from $s=5$ (for $s>5$, we assume that $f_{1}$ is accurately represented by (4.9)) to $s=2$. The value of the dipole strength $f_{1}^{\infty}$ is adjusted until the boundary condition at $s=2$ is achieved. The hydrodynamic functions $G(\hat{b} s), H(\hat{b} s)$ and $W(\hat{b} s)$ for $\hat{b} s>2.01$ are computed via the twin multipole expansion of Jeffrey \& Onishi (1984), whilst for $\hat{b} s<2.01$ the lubrication theory results detailed in Kim \& Karilla (1991) are used. In figure 2, we plot $f_{1}$ versus $s$ for several values of $\hat{b}$.

At $O\left(P e_{b}^{2}\right)$, we obtain a system of equations for $f_{2}$ and $h_{2}$. For $f_{2}$ we have

$$
\begin{aligned}
& \frac{\mathrm{d}}{\mathrm{d} s}\left(s^{2} G(\hat{b} s) \frac{\mathrm{d} f_{2}}{\mathrm{~d} s}\right)-6 H(\hat{b} s) f_{2}=\left(s H(\hat{b} s)-s^{2} W(\hat{b} s)\right) f_{1}-G(\hat{b} s) \frac{\mathrm{d} f_{1}}{\mathrm{~d} s}, \\
& \frac{\mathrm{d} f_{2}}{\mathrm{~d} s}=-f_{1} \text { at } s=2, \\
& f_{2} \rightarrow 0 \text { as } s \rightarrow \infty,
\end{aligned}
$$

and for $h_{2}$

$$
\begin{aligned}
\frac{\mathrm{d}}{\mathrm{d} s}\left(s^{2} G(\hat{b} s) \frac{\mathrm{d} h_{2}}{\mathrm{~d} s}\right) & =-s^{2} H(\hat{b} s) f_{1}-2 s H(\hat{b} s) f_{2}, \\
\frac{\mathrm{d} h_{2}}{\mathrm{~d} s} & =0 \text { at } s=2, \\
h_{2} & \rightarrow 0 \text { as } s \rightarrow \infty .
\end{aligned}
$$




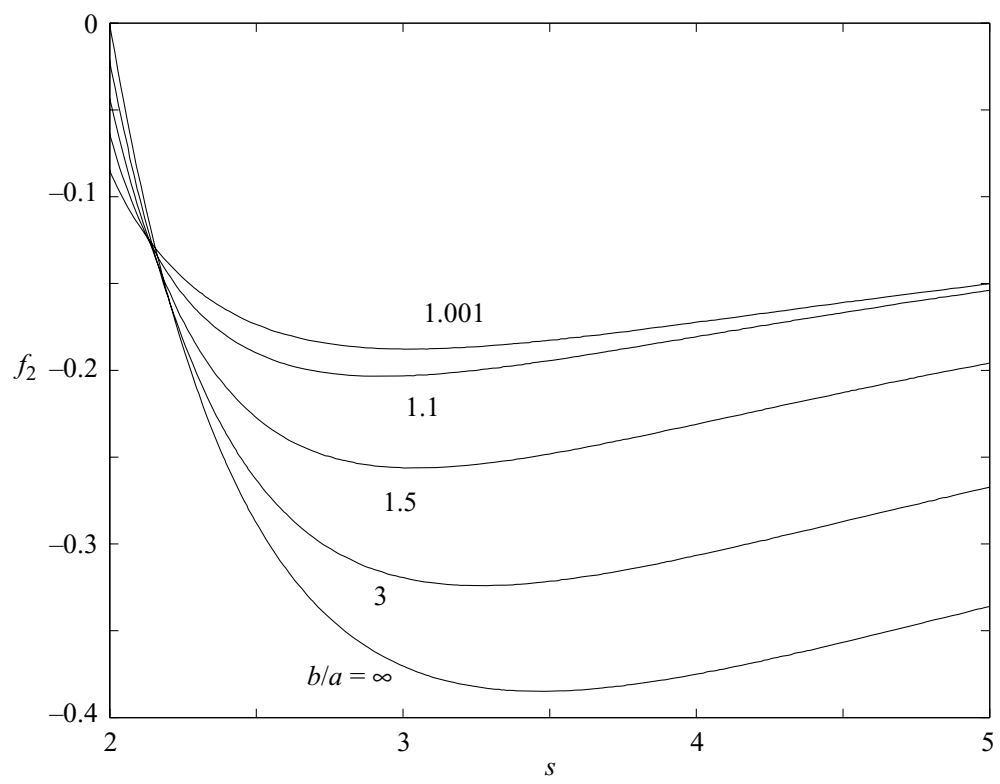

FIGURE 3. First $O\left(P e_{b}^{2}\right)$ structural deformation function $f_{2}$ for several values of $\hat{b}=b / a$.

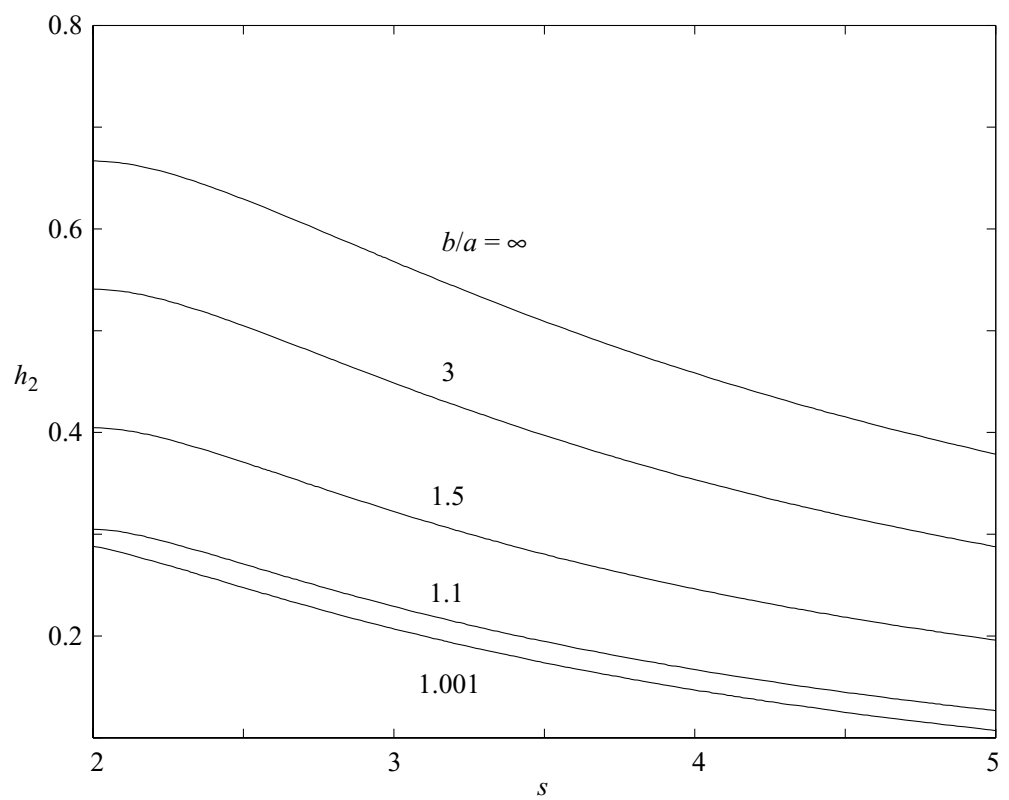

FIGURE 4. Second $O\left(P e_{b}^{2}\right)$ structural deformation function $h_{2}$ for several values of $\hat{b}=b / a$.

To obtain $f_{2}$ and $h_{2}$, a similar procedure is adopted to that in the $f_{1}$ problem; figures 3 and 4 plot $f_{2}$ and $h_{2}$, respectively, versus $s$ for several values of $\hat{b}$.

As mentioned earlier, the $O\left(P e_{b}\right)$ and $O\left(P e_{b}^{2}\right)$ inner solutions will match to the leading-order $O\left(P e_{b}^{3}\right)$ outer solution. At distances $s \sim O\left(P e_{b}^{-1}\right)$ the effects of hydrodynamic interactions may be neglected in as much as the outer solution satisfies (4.2). 
The general solution to (4.2) may be written as (Acrivos \& Taylor 1965)

$$
F=\frac{\pi}{\rho} \exp \left(-\frac{\rho}{2}(1-\mu)\right) \sum_{l=0}^{\infty} A_{l} P_{l}(\mu) \sum_{k=0}^{l} \frac{(l+k) !}{k !(l-k) !} \rho^{-k},
$$

where we have taken $\hat{\boldsymbol{F}}^{\text {ext }}=-\hat{\boldsymbol{z}}, \mu=\cos \theta$, and $P_{l}(\mu)$ is the Legendre polynomial of order $l$ and argument $\mu$. The expansion coefficients $A_{l}$ are found by matching (4.12) to the outer limit of the inner solution, which is

$$
f \sim-P e_{b}^{3} \mu \frac{f_{1}^{\infty}}{\rho^{2}}+P e_{b}^{3} P_{2}(\mu) \frac{2 f_{2}^{\infty}}{3 \rho}+\frac{P e_{b}^{3}}{\rho}\left(\frac{f_{2}^{\infty}}{3}+h_{2}^{\infty}\right),
$$

in terms of the outer variable $\rho$. The scalars $f_{1}^{\infty}, f_{2}^{\infty}$ and $h_{2}^{\infty}$ are coefficients of the leading-order terms of $f_{1}, f_{2}$ and $h_{2}$, respectively, as $s \rightarrow \infty$; hence, the outer solution is indirectly influenced by hydrodynamic interactions. Matching inner and outer solutions, we find that

$$
A_{0}=P e_{b}^{3} \frac{h_{2}^{\infty}}{\pi}, \quad A_{1}=-P e_{b}^{3} \frac{f_{1}^{\infty}}{2 \pi}, \quad A_{l}=0 \quad \forall l>1,
$$

so that the outer solution to leading order is

$$
F=\frac{P e_{b}^{3}}{\rho} \exp \left(-\frac{\rho}{2}(1-\mu)\right)\left\{h_{2}^{\infty}+\mu f_{2}^{\infty}\left(1+\frac{2}{\rho}\right)\right\},
$$

which is basically the Green's function for (4.2) with an additional term (proportional to $f_{2}^{\infty}$ ) accounting for the dipole structure of the leading-order inner solution. Physically, on the scale of $\rho$ the probe appears to be a point source of structural deformation. At large distances $\left(s \sim O\left(P e_{b}^{-1}\right)\right)$ the perturbation to the equilibrium microstructure produced by this point source is exponentially small everywhere except in a wake region where $\rho(1-\mu) \sim O(1)$ in which the decay is algebraic $\sim \rho^{-1}$. In the limit $\rho \rightarrow \infty$, the area of non-zero structural deformation is restricted to $\theta \sim O\left(\rho^{-1 / 2}\right)$.

\subsection{Linear response: the intrinsic microviscosity and its relation to self-diffusivity}

Having analysed the perturbation to the equilibrium microstructure we now proceed to compute the resulting intrinsic microviscosity. First, we shall consider small departures from equilibrium, the so-called linear-response regime, where to $O\left(P e_{b}\right)$ the distortion of the equilibrium microstructure is linearly related to the external force. This is the realm of passive microrheology. In addition to providing a valuable check of our numerical calculations, we can relate the intrinsic microviscosity in this limit to the long-time self-diffusivity.

There are three translational diffusive processes occurring in colloidal dispersions; each characterized with it's own diffusivity: the short-time self-diffusivity $D_{0}^{s}$; the long-time self-diffusivity $D_{\infty}^{s}$; and the collective, or down-gradient, diffusivity $D^{c}$. For an isolated colloidal particle all three diffusivities are identical and equal to the Stokes-Einstein-Sutherland diffusivity $D=k T / 6 \pi \eta a$. However, at finite particle concentrations the three diffusivities are different and correspond to distinct physical processes. Both $D_{0}^{s}$ and $D_{\infty}^{s}$ are concerned with the diffusion of a single test particle in a macroscopically quiescent dispersion (although on quite different time scales), whilst $D^{c}$ is the constant of proportionality relating the flux of particles down a steady small concentration gradient (see e.g. Batchelor 1976).

The short-time self-diffusivity measures the instantaneous, or local, mobility of a particle on a time interval which is large compared to the inertial, or momentum, 
relaxation time scale of the particle $t_{I}=m / 6 \pi \eta a$ (where $m$ is the mass of the particle), but small compared to the diffusive time scale of the particle $t_{D}=a^{2} / D$. Within this time interval the particle will have experienced many collisions from the surrounding solvent molecules without moving an appreciable fraction of its size and hence without affecting the spatial arrangement of the particles surrounding it. Thus, we define the short-time self-diffusivity as the ensemble average of the particle mobility with respect to the equilibrium configuration of the dispersion. If the test particle experiences hydrodynamic interactions with surrounding particles, its mobility will be decreased from its value at infinite dilution; therefore, the short-time self-diffusivity is less than the Stokes-Einstein-Sutherland diffusivity. The difference between the shorttime self-diffusivity and the Stokes-Einstein-Sutherland diffusivity is nothing but the equilibrium hydrodynamic microviscosity $\eta_{i, 0}^{H}$, defined in (3.14) as the ensemble average of the probe particle's mobility with respect to the equilibrium pair-distribution function. Indeed, we may make the formal relationship

$$
\frac{D_{0}^{s}}{D}=1-\eta_{i, 0}^{H} \phi_{b},
$$

correct to first order in $\phi_{b}$ (there is also a direct relation between $D_{0}^{s}$ and $\eta_{i, 0}^{H}$ valid for all $\phi_{b}$, Brady 1994).

To examine the effects of hydrodynamic interactions on $D_{0}^{s}$ we may view $\eta_{i, 0}^{H}$ as a function of the excluded radius $\hat{b}$. In the limit of long-range excluded volume interactions $(\hat{b} \gg 1)$, the asymptotic formulae for $A_{11}$ and $B_{11}$ (see e.g. Kim \& Karilla 1991) may be used to show

$$
\eta_{i, 0}^{H}=\frac{15}{8 \hat{b}^{4}}-\frac{9}{64 \hat{b}^{6}}-\frac{1}{20 \hat{b}^{8}}+\frac{737}{7168 \hat{b}^{10}}+O\left(\hat{b}^{-12}\right) .
$$

For general values of $\hat{b}$, we must evaluate $\eta_{i, 0}^{H}$ numerically. This is accomplished by splitting the range of integration $[2, \infty)$ into three regions: $2 \leqslant \hat{b} s<2.01$, in which the contribution to the integral is evaluated analytically using lubrication theory results for the hydrodynamic functions; $2.01 \leqslant \hat{b} s<3$, where the hydrodynamic functions are obtained via a twin multipole expansion (Jeffrey \& Onishi 1984) and the integral is performed numerically; and $3 \leqslant \hat{b} s<\infty$, where the far-field forms of the hydrodynamic functions are used. In figure 5 we plot $\eta_{i, 0}^{H}$ as a function of $\hat{b}$; the solid line in figure 5 is the first term in the series (4.17), whilst the circles represent the full numerical evaluation. The agreement between the two is excellent. For the case of full hydrodynamic interactions $(\hat{b}=1)$ we find $\eta_{i, 0}^{H}=1.83$ in agreement with Batchelor (1976). Note, the first term in the series (4.17) gives a value of $\eta_{i, 0}^{H}=1.875$ for $\hat{b}=1$, which is remarkably close to the actual value. As $\eta_{i, 0}^{H}$ is positive, the effect of hydrodynamic interactions is to decrease $D_{0}^{s} / D$, as the motion of the probe is hindered by hydrodynamic interactions with its neighbours. Of course, in the limit $\hat{b} \rightarrow \infty$ the mobility of the probe is not affected by the presence of the background particles and $D_{0}^{s} / D \rightarrow 1$, i.e. the probe is isolated (in a hydrodynamic sense) from neighbouring particles.

In contrast, the long-time self-diffusivity corresponds to motion on time scales much greater than the diffusive timescale $t_{D}$, so that the test particle will have experienced many uncorrelated encounters with surrounding particles. As it diffuses, the test particle distorts its local environment from the equilibrium configuration. Brady (1994) has shown that the microstructural evolution problem for self-diffusivity (at long wavelengths) is identical to that for a probe particle moving under the action of a 


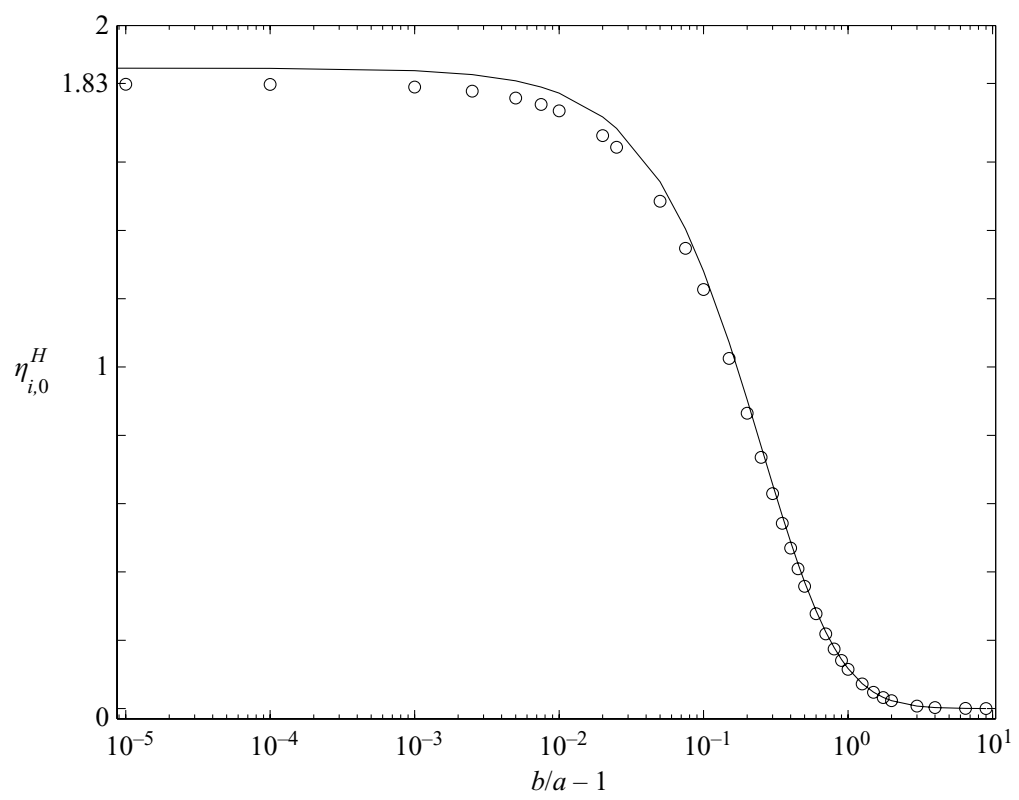

FIGURE 5. The equilibrium microstructure contribution the intrinsic hydrodynamic microviscosity $\eta_{i, 0}^{H}$ as a function of the excluded radius $\hat{b}=b / a$. $\bigcirc$, numerical computations; asymptotic result $\eta_{i, 0}^{H}=15 / 8 \hat{b}^{4}+O\left(1 / \hat{b}^{6}\right)$. Recall, $\eta_{i, 0}^{H}$ is related to the short-time self-diffusivity via $D_{0}^{s} / D-1=-\eta_{i, 0}^{H} \phi_{b}$.

weak external force $\boldsymbol{F}^{\text {ext }}$. In the passive-microrheology (or linear-response) regime the distortion to the equilibrium microstructure is linear in $\boldsymbol{F}^{\text {ext }}$. Consequently, the average velocity of the probe $\langle\boldsymbol{U}\rangle$ is proportional to $\boldsymbol{F}^{\text {ext }}$, with the constant of proportionality being the time-dependent self-diffusivity $D^{s}(t)$ divided by $k T$. At long times $\left(t \gg t_{D}\right), D^{s}(t) \rightarrow D_{\infty}^{s}$, and we recover the long-time self-diffusivity. In the linear response regime the distorted microstructure is given by $P e_{b} f_{1}(s) \hat{\boldsymbol{s}} \cdot \hat{\boldsymbol{F}}^{e x t}$. This $O\left(P e_{b}\right)$ deformation of the microstructure leads to an $O(1)$ contribution to the interparticle and Brownian microviscosities as seen from (3.15) and (3.16), respectively, which we denote temporarily as $\eta_{i, 0}^{P}$ and $\eta_{i, 0}^{B}$, respectively. The long-time self-diffusivity may then be expressed as

$$
\frac{D_{\infty}^{s}}{D}=1-\eta_{i, 0} \phi_{b}
$$

where $\eta_{i, 0}=\eta_{i, 0}^{H}+\eta_{i, 0}^{P}+\eta_{i, 0}^{B}$, and $\eta_{i, 0}^{P}$ and $\eta_{i, 0}^{B}$ are given by

$$
\begin{aligned}
& \eta_{i, 0}^{P}=2 G(2 \hat{b}) f_{1}(2), \\
& \eta_{i, 0}^{B}=\int_{2}^{\infty}\left(\frac{G(\hat{b} s)-H(\hat{b} s)}{s}+\frac{1}{2} \frac{\mathrm{d} G(\hat{b} s)}{\mathrm{d} s}\right) f_{1}(s) s^{2} \mathrm{~d} s .
\end{aligned}
$$

In figure 6, we plot $\eta_{i, 0}$ as a function of $\hat{b}$. At the extrema of $\hat{b}=1$ (full hydrodynamic interactions) and $\hat{b} \rightarrow \infty$ (no hydrodynamic interactions) we find that $\eta_{i, 0}=2.08$ and $\eta_{i, 0}=2$, respectively, in good agreement with Batchelor (1976) and Rallison \& Hinch (1986). As $\hat{b}$ is increased, hydrodynamic interactions become weaker; hence, $\eta_{i, 0}^{H}$ and $\eta_{i, 0}^{B}$ are monotonically decreasing functions of $\hat{b}$ (recall that the effect of Brownian 


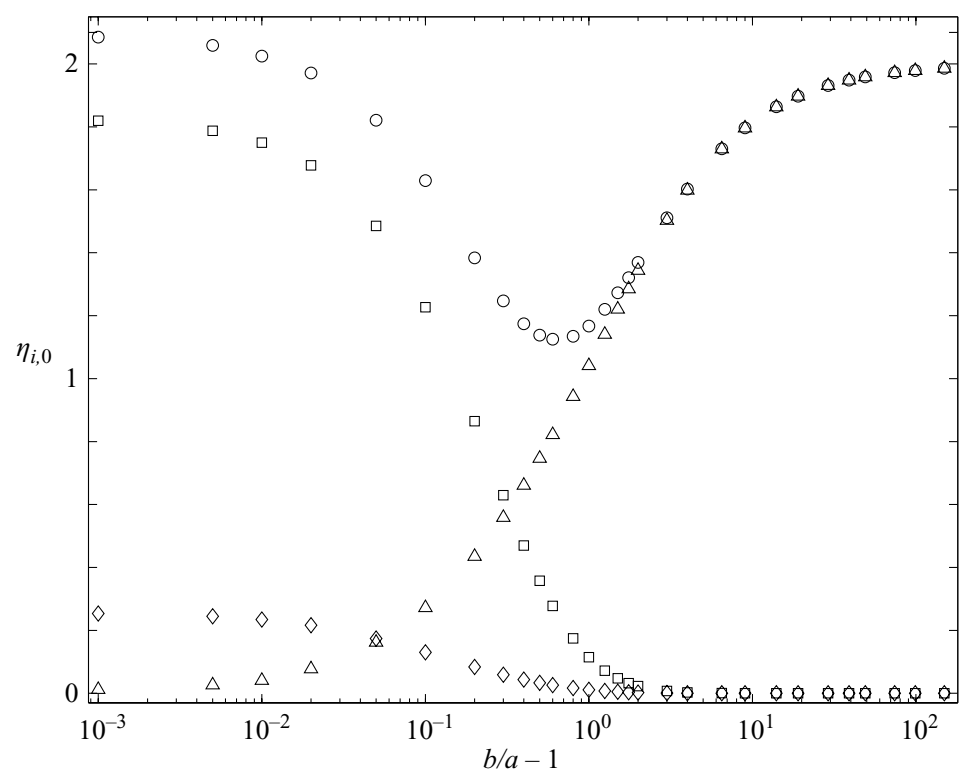

FIGURE 6. Intrinsic microviscosity contributions in the limit $P e_{b} \rightarrow 0$ as a function of $\hat{b}=b / a$ : $\bigcirc$, total $\left(\eta_{i, 0}\right) ; \square$, hydrodynamic $\left(\eta_{i, 0}^{H}\right) ; \diamond$, Brownian $\left(\eta_{i, 0}^{B}\right) ; \Delta$, interparticle force $\left(\eta_{i, 0}^{P}\right)$. Recall, $\eta_{i, 0}$ is related to the long-time self-diffusivity via $D_{\infty}^{s} / D-1=-\eta_{i, 0} \phi_{b}$.

motion on the velocity of the probe particle appears as a hydrodynamic coupling of the motion of two particles, see (3.10)). The decrease in $\eta_{i, 0}^{H}$ and $\eta_{i, 0}^{B}$ is offset by an increase in the interparticle microviscosity $\eta_{i, 0}^{P}$, from a value of $\eta_{i, 0}^{P}=8(\hat{b}-1) f_{1}(2)$ in the limit $\hat{b} \rightarrow 1$ to $\eta_{i, 0}^{P}=2$ as $\hat{b} \rightarrow \infty$. The decay of $\eta_{i, 0}^{H}$ and $\eta_{i, 0}^{B}$ for $\hat{b}$ slightly above unity cannot be matched by the relatively small increase in $\eta_{i, 0}^{P}$; thus, $\eta_{i, 0}$ is an initially decreasing function of $\hat{b}$. This decrease persists until $\hat{b} \approx 1.6$ where $\eta_{i, 0}$ exhibits a minimum. Beyond $\hat{b} \approx 1.6$ there is a monotonic increase of $\eta_{i, 0}$ to its limiting value of 2 as $\hat{b} \rightarrow \infty$.

The non-monotonicity of $\eta_{i, 0}$ is somewhat surprising and suggests the intriguing possibility of maximizing the long-time self-diffusivity of a particle through modulation of its interparticle-force interactions. If the volume fraction in (4.18) were based on the hydrodynamic radius $a$ instead of the thermodynamic radius $b$ (note that $\phi_{b}=$ $\hat{b}^{3} \phi_{a}$ ), we must multiply $\eta_{i, 0}$ by $\hat{b}^{3}$, implying that the long-time self-diffusivity decreases monotonically (and without bound) with increasing $\hat{b}$. However, the geometric, or excluded, radius $b$ is the correct length scale in defining the volume fraction, as particles must actually move past each other on this scale. The maximum in the long-time self-diffusivity (at $\hat{b} \approx 1.6$ ) arises because $\hat{b}$ is sufficiently greater than unity, so that the highly resistive hydrodynamic lubrication interactions do not hinder the motion of the probe, while $\hat{b}$ is not too large, whence the long-time self-diffusivity decreases owing to the increased role of the hard-sphere interparticle-force. Although the values of long-time self-diffusivity at the extrema of $\hat{b}=1$ and $\hat{b} \rightarrow \infty$ are similar, it is interesting to contrast the physical mechanisms at work in both limits: for $\hat{b}=1$, the resistance to the probe motion is via hydrodynamic interactions with other particles, which are mediated through the solvent fluid, whilst in the limit $\hat{b} \rightarrow \infty$, the probe motion is hindered by the excluded volume interparticle-force, which acts at contact to provide a purely geometric resistance to the probe motion. 


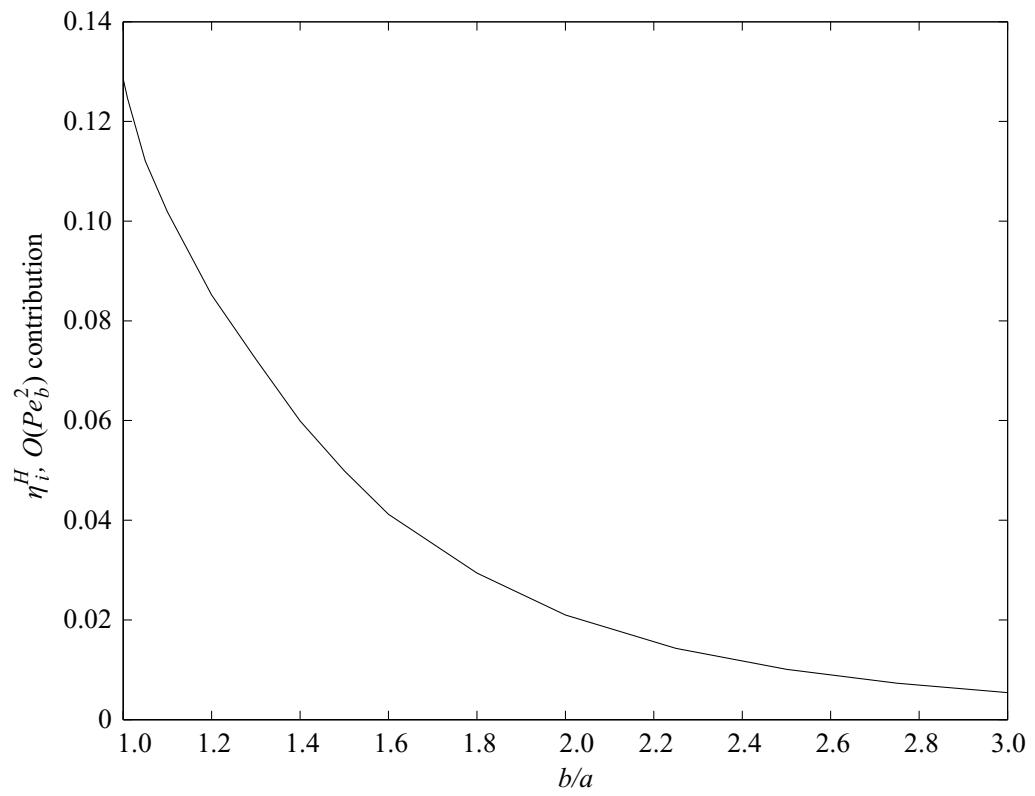

Figure 7. The $O\left(P e_{b}^{2}\right)$ contribution to the intrinsic hydrodynamic microviscosity $\eta_{i}^{H}(4.20)$ as a function of $\hat{b}=b / a$.

\subsection{Weakly nonlinear theory}

Our discussion of the intrinsic microviscosity and its relation to self-diffusivity took place in the regime of linear response, where the departure from the equilibrium microstructure is small and linearly related to the external force and $P e_{b}$. The linear relationship between the distorted microstructure and the external force manifests itself in the structural deformation being fore-aft symmetric about the direction of the external force. Upon increasing $P e_{b}$, we enter the nonlinear regime where the distortion to the equilibrium microstructure is no longer linearly related to $P e_{b}$, and the fore-aft symmetry of the structural deformation about the external force is broken. The first nonlinear contribution to the structural deformation (in the inner region) occurs at $O\left(P e_{b}^{2}\right)$ and was calculated in $\S 4.1$. Here, we compute the effect on the intrinsic microviscosity arising from this $O\left(P e_{b}^{2}\right)$ nonlinear deformation.

First, it is readily seen from (4.7) and the symmetry of the integrals in (3.15) and (3.16) that the $O\left(P e_{b}^{2}\right)$ structural deformation does not contribute to the Brownian and interparticle microviscosities; however, there is a contribution to the hydrodynamic microviscosity, which is given by

$\eta_{i}^{H}-\eta_{i, 0}^{H}=-\frac{P e_{b}^{2}}{5}\left(\int_{2}^{\infty}\left(3 A_{11}+2 B_{11}-5\right) f_{2}(s) s^{2} \mathrm{~d} s+\int_{2}^{\infty}\left(5 A_{11}+10 B_{11}-15\right) h_{2}(s) s^{2} \mathrm{~d} s\right)$,

and is evidently of $O\left(P e_{b}^{2}\right)$. This $O\left(P e_{b}^{2}\right)$ contribution is plotted in figure 7 as a function of $\hat{b}$ and is seen to be positive for finite $\hat{b}$ and approaches zero as $\hat{b} \rightarrow \infty$. Hence, the hydrodynamic microviscosity is an increasing function of $P e_{b}$, for small $P e_{b}$ up to $O\left(P e_{b}^{2}\right)$. Bergenholtz et al. (2002) observed that the hydrodynamic macroviscosity of a dilute sheared suspension is an increasing function for all $P e_{b}$, i.e. the hydrodynamic macroviscosity monotonically 'shear-thickens'. Our results indicate the hydrodynamic 
microviscosity undergoes an analogous 'force-thickening'. The persistence of this force-thickening for larger values of $P e_{b}$ will be verified by the numerical calculations presented in $\S 6$.

The microviscosity contributions presented above are all from the inner region, where $s \sim O(1)$, and we must also consider the magnitude of the contributions arising from the outer region, where $s \sim O\left(\rho P e_{b}^{-1}\right)$. At large distances, the non-equilibrium contribution to the hydrodynamic microviscosity behaves as

$$
\eta_{i}^{H}-\eta_{i, 0}^{H} \sim \int \frac{1}{s^{4}} f s^{2} \mathrm{~d} s \sim O\left(P e_{b}^{4}\right),
$$

since $f \sim O\left(P e_{b}^{3}\right)$ in the outer region. Hence, the $O\left(P e_{b}^{2}\right)$ contribution to $\eta_{i}^{H}$ comes exclusively from the inner region. Similarly, the outer contribution to the Brownian microviscosity is

$$
\eta_{i}^{B} \sim \frac{1}{P e_{b}} \int \frac{1}{s^{5}} f s^{2} \mathrm{~d} s \sim O\left(P e_{b}^{4}\right) .
$$

However, the next term in the inner solution, $P e_{b}^{3} f_{3}$, will generate an $O\left(P e_{b}^{2}\right)$ contribution to $\eta_{i}^{B}$ (and to $\eta_{i}^{P}$ ) and is therefore of lower order than the $O\left(P e_{b}^{4}\right)$ contribution from the leading-order outer solution.

\section{Numerical solution of the Smoluchowski equation for arbitrary $\boldsymbol{P e}_{b}$}

The perturbation analysis presented above sheds light on the microstructural deformation in the case of small departures from equilibrium. Attempting to continue the expansion to higher orders in $P e_{b}$ is unwise as the matching of inner and outer solutions must be performed numerically (except in the absence of hydrodynamic interactions where it can be done analytically) and the algebra involved becomes rapidly intractable. Thus, to obtain the microstructure for arbitrary values of $P e_{b}$, we must solve the Smoluchowski equation numerically. For $P e_{b} \sim O(1)$ and higher, this is a challenging task owing to the formation of a boundary layer in the compressional region (where $\hat{\boldsymbol{F}}^{\text {ext }} \cdot \hat{\boldsymbol{s}}>0$ ) around the probe particle and a wake in the extensional region (where $\hat{\boldsymbol{F}}^{\text {ext }} \cdot \hat{\boldsymbol{s}}<0$ ), cf. figure 1 . In figure 8 , we plot the structural deformation in the symmetry plane of the probe particle as a function of $P e_{b}$ for $\hat{b}=1.00001$. The formation of a boundary layer is clearly visible, as is the growth of the wake with increasing $P e_{b}$. In the compressional region there is an inward radial flux of background particles from upstream towards the probe particle (in a frame moving with the probe) owing to advection by the relative velocity field. This flux of particles is hindered by the impenetrability of the probe, resulting in an increased probability of finding a particle in close proximity to the probe particle. The primary mechanism for a particle to pass by the probe is via Brownian diffusion whose action as $P e_{b}$ is increased is confined to an ever smaller boundary layer adjacent to the surface of the probe in the compressional region. The boundary layer signifies a balance between advection in transporting particles towards the probe and Brownian motion in enabling particles to diffuse around it. On the other hand, in the extensional region, the action of the relative velocity field is to advect particles away from the probe, resulting in a decrease in the probability of finding a colloidal particle there.

To solve the Smoluchowski equation for arbitrary $P e_{b}$ we use two methods. For $0<P e_{b} \leqslant 30$, an expansion of the structural deformation in a series of Legendre polynomials is employed, similar in ethos to the spherical harmonic expansions of Bergenholtz et al. (2002) and Lionberger (1998). The major limitation of the Legendre 

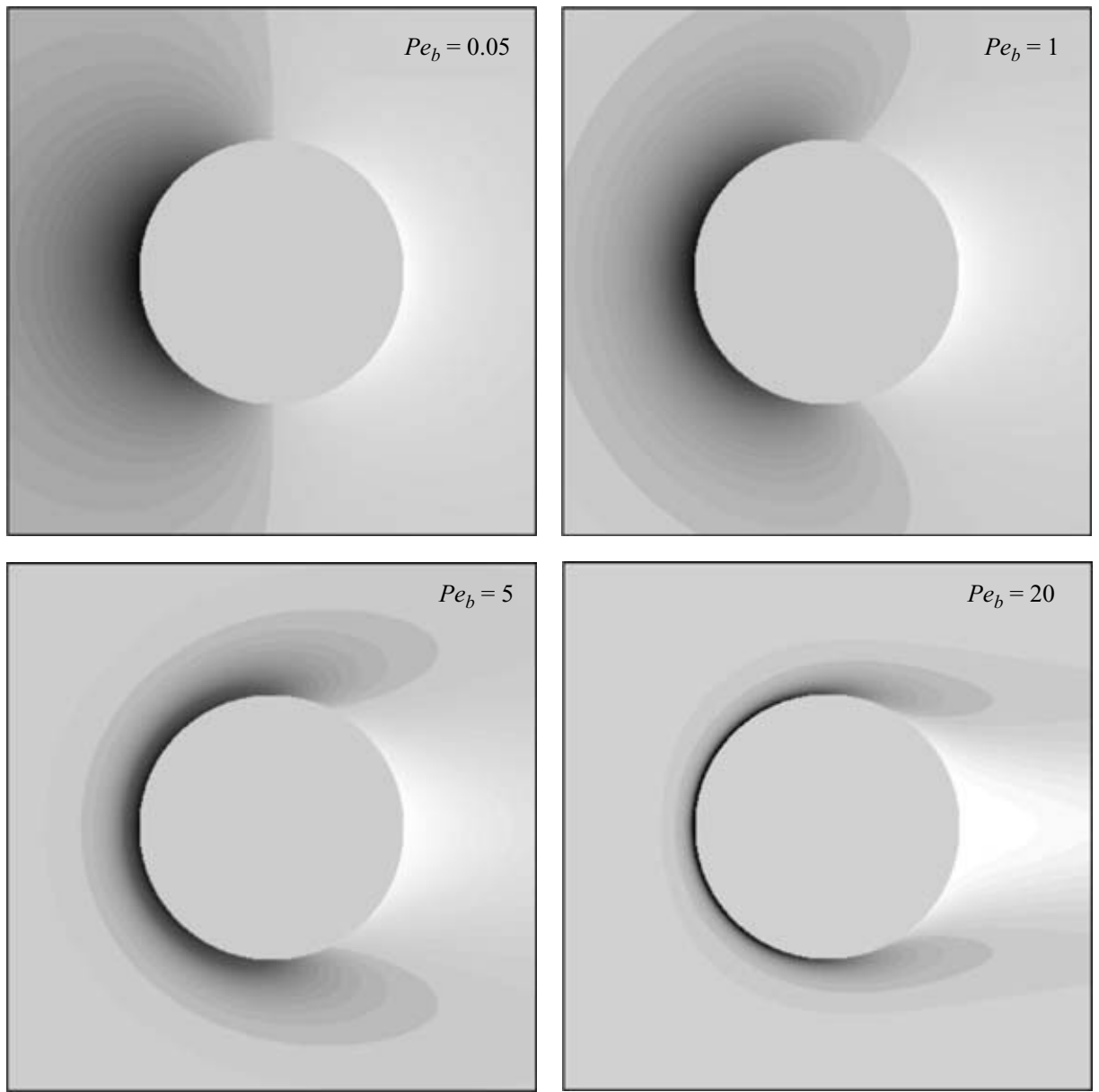

FiguRE 8 . Structural deformation $f(s)=g(s)-1$ in the symmetry plane of the probe particle as a function of $P e_{b}$ for $\hat{b}=1.00001$. The external force $\boldsymbol{F}^{\text {ext }}$ is from right-to-left. The test particle is shown with zero deformation $f=0$, darker regions have positive $f$ while lighter regions have negative $f$.

polynomial expansion is that as $P e_{b}$ is increased, a large number of terms in the expansion are required to represent faithfully the increasingly intricate microstructure, which is computationally taxing. Thus, to solve the Smoluchowski equation for $P e_{b}>30$, we use a finite-difference scheme which accurately captures the boundarylayer formation and wake growth.

\subsection{Legendre polynomial expansion}

We adopt a spherical polar coordinate system with origin at the centre of the probe particle (cf. figure 1). In this coordinate system the Smoluchowski equation for the structural deformation function reads

$$
\begin{aligned}
\frac{1}{s^{2}} & \frac{\partial}{\partial s}\left(s^{2} G(\hat{b} s) \frac{\partial f}{\partial s}\right)+\frac{H(\hat{b} s)}{s^{2} \sin \theta} \frac{\partial}{\partial \theta}\left(\sin \theta \frac{\partial f}{\partial \theta}\right) \\
& =-P e_{b}\left(G(\hat{b} s) F_{r}^{e x t} \frac{\partial f}{\partial s}+H(\hat{b} s) \frac{F_{\theta}^{e x t}}{s} \frac{\partial f}{\partial \theta}+W(\hat{b} s) F_{r}^{e x t}(1+f)\right)
\end{aligned}
$$


where $F_{r}^{e x t}=\hat{\boldsymbol{F}}^{\text {ext }} \cdot \hat{\boldsymbol{s}}$ and $F_{\theta}^{\text {ext }}=\hat{\boldsymbol{F}}^{\text {ext }} \cdot \hat{\boldsymbol{\theta}}$ are the radial and polar components of the external force unit vector, respectively. The boundary conditions on $f(s, \theta)$ are

$$
\begin{aligned}
& \frac{\partial f}{\partial s}=-P e_{b} F_{r}^{e x t}(1+f) \quad \text { at } \quad s=2, \\
& f \rightarrow 0 \text { as } s \rightarrow \infty \text {. }
\end{aligned}
$$

The structural deformation may be written as an expansion in Legendre polynomials

$$
f(s, \theta)=\sum_{m=0}^{\infty} q_{m}(s) P_{m}(\cos \theta),
$$

where $P_{m}(z)$ is the Legendre polynomial of order $m$ and argument $z$ and $q_{m}(s)$ its expansion coefficient. The expansion is substituted into (5.1) and upon use of the orthogonality property of the Legendre polynomials on the interval $[0, \pi]$, we arrive at an infinite set of coupled ordinary differential equations for the expansion coefficients. Additionally, if we assume (without loss of generality) that $\hat{\boldsymbol{F}}^{\text {ext }}=-\hat{\boldsymbol{z}}$, so that $F_{r}^{e x t}=-\cos \theta$ and $F_{\theta}^{e x t}=\sin \theta$, the set of equations takes the form

$$
\mathscr{D}_{m} q_{m}=P e_{b}\left(W(\hat{b} s) \alpha_{m}+G(\hat{b} s) \beta_{m}+\frac{H(\hat{b} s)}{s} \gamma_{m}\right),
$$

where the diffusion operator $\mathscr{D}_{m}$ is

$$
\mathscr{D}_{m}=\frac{1}{s^{2}} \frac{\mathrm{d}}{\mathrm{d} s}\left(s^{2} G(\hat{b} s) \frac{\mathrm{d}}{\mathrm{d} s}\right)-\frac{H(\hat{b} s) m(m+1)}{s^{2}},
$$

and the advective coupling terms $\alpha_{m}, \beta_{m}$ and $\gamma_{m}$ are

$$
\begin{aligned}
\alpha_{m} & =\frac{2 m+1}{3} \delta_{m 1}+\frac{m+1}{2 m+3} q_{m+1}+\frac{m}{2 m-1} q_{m-1}, \\
\beta_{m} & =\frac{m+1}{2 m+3} \frac{\mathrm{d} q_{m+1}}{\mathrm{~d} s}+\frac{m}{2 m-1} \frac{\mathrm{d} q_{m-1}}{\mathrm{~d} s}, \\
\gamma_{m} & =\frac{(m+1)(m+2)}{(2 m+3)} q_{m+1}+\frac{m(1-m)}{2 m-1} q_{m-1},
\end{aligned}
$$

with $\delta_{i j}$ the Kronecker delta. The boundary conditions on the expansion coefficients are

$$
\begin{aligned}
& \frac{\mathrm{d} q_{m}}{\mathrm{~d} s}=P e_{b}\left(\frac{2 m+1}{3} \delta_{m 1}+\frac{m+1}{2 m+3} q_{m+1}+\frac{m}{2 m-1} q_{m-1}\right) \quad \text { at } \quad s=2 \text {, } \\
& q_{m} \rightarrow 0 \quad \text { as } \quad s \rightarrow \infty \text {. }
\end{aligned}
$$

The system of equations is solved with the MATLAB program $b v p 4 c$, which implements a collocation method for the solution of general two-point boundary-value problems. An initial guess for each of the expansion coefficients on a user-defined mesh covering the domain of solution is provided as input to the program, which subsequently refines the mesh to obtain the numerical solution to a preset accuracy (usually specified in terms of the absolute tolerance). A notable feature of the bvp $4 c$ routine is the ability to perform parameter continuation, i.e. suppose that one has the structural deformation at some $P e_{b}=P e_{b, 1}$ then this solution may be used as the initial guess for the structural deformation at $P e_{b}=P e_{b, 2}>P e_{b, 1}$. This is particularly useful for $P e_{b}>10$, where the large gradients in the structural deformation encountered in the boundary layer make the choice of initial guess crucial to the convergence of the method. The expansion is truncated at $m=m_{\max }$ so that $q_{m}=0 \forall m>m_{\max }$. The choice of $m_{\max }$ for a particular $P e_{b}$ is made by requiring that each of the three contributions 
to the intrinsic microviscosity should not differ by more than $0.1 \%$ when computed using $m_{\max }$ and $m_{\max +1}$ terms. As $P e_{b}$ increases so does $m_{\max }$, reflecting the need for more terms in the expansion to describe the microstructure accurately. The highest value of $P e_{b}$ for which a solution was obtained was $P e_{b}=30$ requiring $m_{\max }=60$.

Finally, some care needs to be taken in application of the far-field boundary condition. Here, we make the simple approximation of moving the boundary condition at infinity to a finite radial location $s=s_{f a r}$, taking great care to ensure that the choice of $s_{f a r}$ does not affect the computed intrinsic microviscosity contributions. For $P e_{b}<1$ at radial distances $O\left(P e_{b}^{-1}\right)$, advection is comparable to diffusion, requiring $s_{f a r} \gg O\left(P e_{b}^{-1}\right)$. As a starting point, $s_{f a r}$ is chosen to be $10^{3} P e_{b}^{-1}$ and increased until convergence of each of the intrinsic microviscosity contributions to eight decimal places is achieved. For $P e_{b}>1$, where, in addition to the boundary-layer structure at the front of the probe, we must also account for the wake region behind it (whose characteristic length grows linearly with $P e_{b}$ ), we start with $s_{f a r}=10^{2} P e_{b}$ and increase $s_{f a r}$ until the intrinsic microviscosity contributions converge. In $\S 6$, several studies are presented that validate our approximation of the far-field boundary condition and demonstrate the accuracy of the numerical solutions.

\subsection{Finite-difference methods}

Numerically solving the Smoluchowski equation accurately at large $P e_{b}$ is a demanding task: the challenge is to capture the detailed boundary-layer structure of the pairdistribution function near contact whilst maintaining sufficient resolution in the farfield to represent the growing wake region behind the probe. In the range $2 \leqslant P e_{b} \leqslant$ 100, the Smoluchowski equation (5.1) is approximated by a finite-difference equation, on a two-dimensional grid which has a dense collection of nodes in the boundary layer (to capture the large gradients in the pair-distribution function there) and nodes distributed sparsely in the far-field. Both radial and angular derivatives are approximated via central differences. Discretization of the Smoluchowski equation leads to the linear matrix equation $\boldsymbol{A} \cdot \boldsymbol{f}=\boldsymbol{w}$, where the coefficient matrix $\boldsymbol{A}$ is tridiagonal with two fringes, $\boldsymbol{f}$ is the unknown structural deformation vector, and $\boldsymbol{w}$ is the forcing vector. The matrix equation is solved iteratively using a simple Jacobi scheme, requiring computation of the inverse of the tridiagonal portion of $\boldsymbol{A}$, which is performed via a standard back-substitution algorithm (Press et al. 1992). The method is efficient in the sense that only the inverse of a tridiagonal matrix is to be computed, but inefficient (as compared to other iterative techniques such as Gauss-Seidel or SOR) as it requires a large number of iterations, to converge. To reduce the number of iterations we employ the convergence acceleration scheme proposed by $\mathrm{Ng}$ (1974). A detailed exposition of the finite-difference method is provided in Appendix B.

As $P e_{b}$ is increased, we require a greater number of grid points (and hence iterations) for the method to converge. Beyond $P e_{b} \approx 100$, the iteration diverges; at such large $P e_{b}$ it is reasonable to postulate that the rheological properties of the suspension are primarily determined by the large gradients in the pair-distribution function occurring in the boundary layer. Thus, for $P e_{b}>100$, we solve a boundary-layer approximation to the full Smoluchowski equation, which is derived in Appendix C. This equation retains the information on the detailed structure of the boundary layer at the expense of obtaining accurate far-field behaviour. The boundary-layer equation reads

$G \frac{\partial^{2} f}{\partial y^{2}}+Q \frac{\partial f}{\partial y}+\frac{P e_{b}^{-2} H}{4 \sin \theta} \frac{\partial}{\partial \theta}\left(\sin \theta \frac{\partial f}{\partial \theta}\right)=P e_{b}^{-1}\left(W \cos \theta(1+f)-\frac{1}{2} H \sin \theta \frac{\partial f}{\partial \theta}\right)$,

where $y=P e_{b}(s-2)$ is a stretched radial coordinate, $Q=-G \cos \theta+\mathrm{d} G / \mathrm{d} y+$ $P e_{b}^{-1} G\left(1-P e_{b}^{-1} y / 2\right)$, and terms of $O\left(P e_{b}^{-3}\right)$ and smaller have been discarded. The 


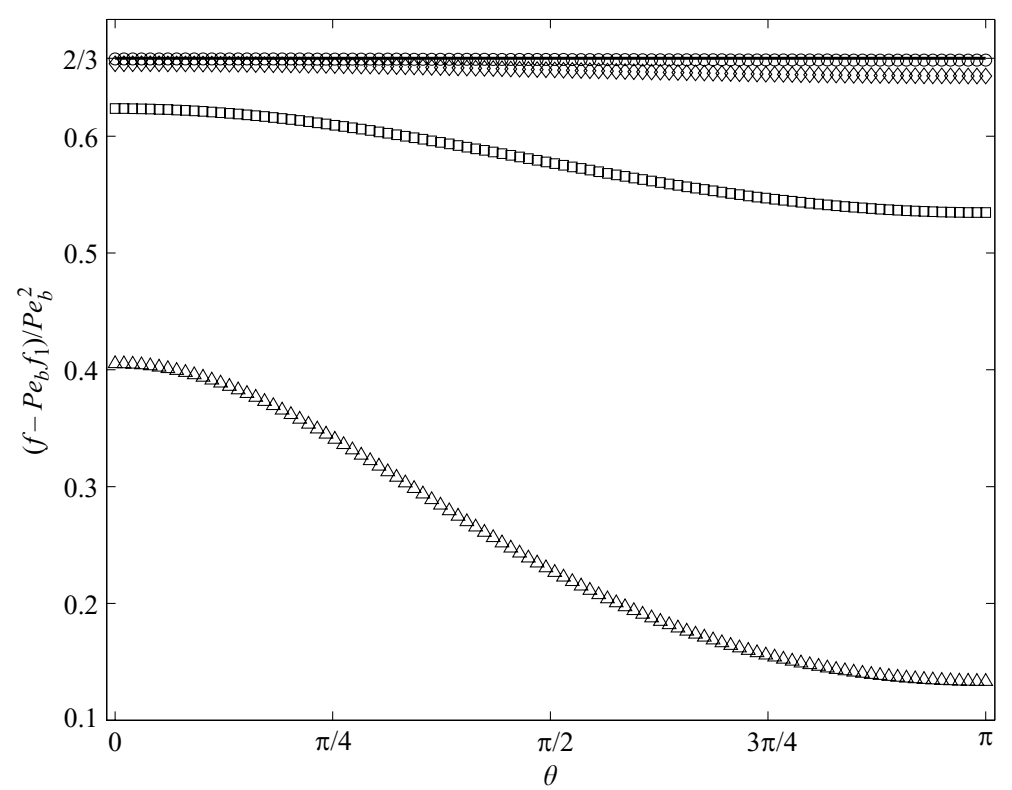

Figure 9. Angular dependence of the structural deformation at contact $(s=2)$ for several $P e_{b}$ in the absence of hydrodynamic interactions: $P e_{b}=10^{-3}(\bigcirc) ; P e_{b}=10^{-2}(\diamond) ; P e_{b}=10^{-1}(\square)$; and $P e_{b}=1(\triangle)$. The solid line is the $O\left(P e_{b}^{2}\right)$ perturbation result $\left(f-P e_{b} f_{1}\right) / P e_{b}^{2}=2 / 3+O\left(P e_{b}\right)$.

crucial distinction between (5.8) and the full Smoluchowski equation (5.1) is the absence of the $1 / s^{2}$ factor multiplying the diffusive terms on the left-hand side of (5.8). This results in the exaggeration of diffusive effects at large $s$. Consequently, although there remains a wake behind the probe particle, its size is diminished, making numerical solution of (5.8) considerably easier than that of (5.1). Strictly speaking, the structural deformation determined from (5.8) should be matched to an outer solution from the advectively dominated region. However, this is not a simple task as the radial matching length is a function of the polar angle $\theta$. Here, we assume that the solution of (5.8) is valid throughout the entire domain, specifically requiring that the solution should vanish at large radial separations. To solve the boundary-layer equation, we use a finite-difference method analogous to that employed for solution of the full Smoluchowski equation.

\section{Results}

\subsection{No hydrodynamic interactions}

In this subsection, the microstructure and microviscosity of the dispersion are examined in the absence of hydrodynamic interactions, $\hat{b} \rightarrow \infty$. Particular attention is paid to this limit as the accuracy of our numerical calculations may be demonstrated via comparison to the exact solution of the Smoluchowski equation derived by Squires $\&$ Brady (2005) and the perturbation analysis of $\S 4$.

For weak forcing, it was shown in $\S 4$ that to $O\left(P e_{b}^{2}\right)$ the contact value of the structural deformation is

$$
f(2, \theta)=-P e_{b} \cos \theta f_{1}(2)+P e_{b}^{2}\left(\cos ^{2} \theta f_{2}(2)+h_{2}(2)\right),
$$

where for definiteness it is assumed $\hat{\boldsymbol{F}}^{\text {ext }}=-\hat{\boldsymbol{z}}$. Figure 9 compares the contact value obtained via the Legendre polynomial expansion to the perturbation theory result. In figure 9, the $O\left(P e_{b}^{2}\right)$ term has been isolated by plotting $\left(f(2, \theta)+P e_{b} \cos \theta\right) / P e_{b}^{2}$ versus 


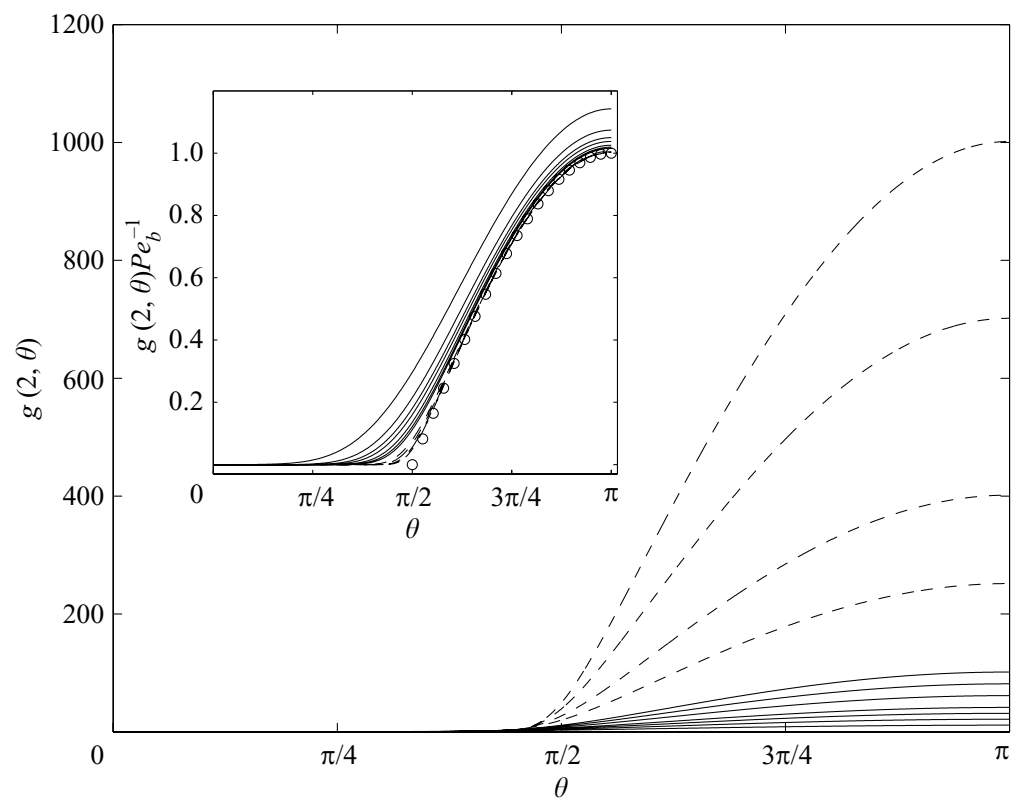

FIGURE 10. The pair-distribution function at contact as a function of the polar angle $\theta$ and $P e_{b}$ in the absence of hydrodynamic interactions, $\hat{b} \rightarrow \infty$. Results from the solution of the full Smoluchowski equation are shown as solid lines whilst solutions from the boundary-layer equation are displayed as broken lines. From bottom-to-top: $P e_{b}=10,20,30,40,60,80$ and 100; and from the boundary-layer equation $P e_{b}=250,400,700$ and 1000. The inset displays the same data scaled by $P e_{b}$ with the addition of the asymptotic result $g(2, \theta) P e_{b}^{-1}=-\cos \theta+$ $O\left(P e_{b}^{-1}\right)(\bigcirc)$ (see Appendix D) valid in the limit $P e_{b} \rightarrow \infty$.

$P e_{b}$ and comparing to the perturbation result $2 / 3+O\left(P e_{b}\right)$. Evidently, the numerical calculations are in good agreement with the perturbation theory up to $P e_{b}=0.1$, beyond which we require higher-order terms in the expansion (6.1) to accurately represent the microstructure.

We now turn our attention to the structural deformation at large $P e_{b}$, which is computed via a finite-difference solution of the full Smoluchowski equation $(2 \leqslant$ $\left.P e_{b} \leqslant 100\right)$ and a boundary-layer approximation of it $\left(20 \leqslant P e_{b} \leqslant 1000\right)$. In the absence of hydrodynamic interactions, there is no reduction in the relative mobility as a forcefree particle approaches the probe particle. Thus, at large $P e_{b}$, the strong inward advective flux of force-free particles (in a reference frame moving with the probe) in the compressional region (upstream) leads to a large accumulation of pair density in a boundary layer at the surface of the probe particle. The probe acts as an obstruction around which the force-free particles coming from upstream must pass. The primary mechanism of passing is via Brownian diffusion, which is driven by the large gradients of pair density present in the boundary layer. In the extensional region (downstream) advection carries the force-free particles away from the probe and a decrease in pair density, or wake, resides there. In Appendix D we show that at large $P e_{b}$ the pair-distribution function is given by

$$
g(s, \theta)= \begin{cases}F_{r}^{e x t} P e_{b} \exp \left\{-F_{r}^{e x t} P e_{b}(s-2)\right\}+O(1) & \text { if } F_{r}^{\text {ext }} \geqslant 0 \\ 0 & \text { if } F_{r}^{\text {ext }}<0\end{cases}
$$

The contact value of the pair-distribution function in the compressional region, $g(2)=$ $F_{r}^{e x t} P e_{b}$, scales linearly with $P e_{b}$. In figure 10, we plot the contact value as a function 


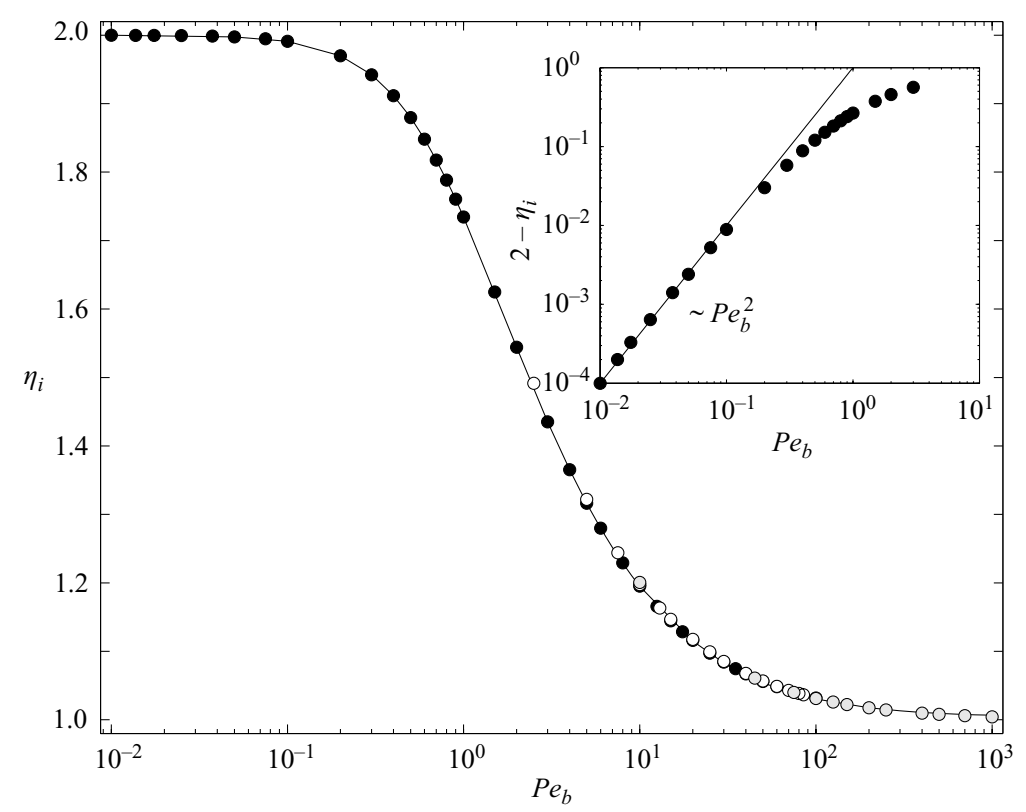

FIGURE 11. The intrinsic microviscosity $\eta_{i}$ as a function of $P e_{b}$ in the absence of hydrodynamic interactions. $\bullet$, Legendre polynomial expansion; $\bigcirc$, finite-difference solution of full Smoluchowski equation; $O$, finite-difference solution of boundary-layer equation. The solid line is the intrinsic microviscosity computed from the exact solution of the Smoluchowski equation by Squires \& Brady (2005). The inset shows that the initial microviscosity variation is proportional to $P e_{b}^{2}$.

of the polar angle $\theta$ for several $P e_{b}$. The inset shows that scaling $g(2)$ with $P e_{b}$ collapses that data well, verifying the linear scaling predicted by (6.2).

In the absence of hydrodynamic interactions the intrinsic microviscosity of the suspension, $\eta_{i}$, is determined solely by the interparticle-force contribution (3.15), which is proportional to the contact value of the structural deformation. Figure 11 plots $\eta_{i}$ as a function of $P e_{b}$. In the limit $P e_{b} \rightarrow 0$, where the structural deformation is linear in the external force, there is a Newtonian plateau at which $\eta_{i}=2$. On increasing $P e_{b}$, the non-Newtonian character of the dispersion is evident in the decrease, or force-thinning, of $\eta_{i}$. The inset reveals that the initial portion of the force thinning is proportional to $P e_{b}^{2}$, arising from the $O\left(P e_{b}^{3}\right)$ structural deformation, $P e_{b}^{3} f_{3}$, which via (3.15) produces an $O\left(P e_{b}^{2}\right)$ contribution to the microviscosity. For $P e_{b} \gg 1$, the microviscosity exhibits a Newtonian plateau with $\eta_{i}$ slightly above unity (for $P e_{b}=10^{3}$ we compute $\eta_{i}=1.004$ ). The boundary-layer analysis in Appendix D predicts $\eta_{i}=1$ at infinite $P e_{b}$. The intrinsic microviscosity calculated by Squires \& Brady (2005) using the exact solution of the Smoluchowski equation is in quantitative agreement with our numerical calculations over the entire range of $P e_{b}$ studied.

\subsection{The effect of hydrodynamic interactions}

Having investigated in detail the special case of particles without hydrodynamic interactions, we now consider the effect of hydrodynamic interactions on the microstructure and microrheology of the dispersion, starting at small $P e_{b}$.

In the limit $P e_{b} \rightarrow 0$, the intrinsic microviscosity for $\hat{b}=1.00001$ is calculated as $\eta_{i}=$ 2.084 , being comprised of the $O\left(\phi_{b}\right)$ short-time self-diffusivity coefficient $\eta_{i, 0}^{H}=1.828$ 


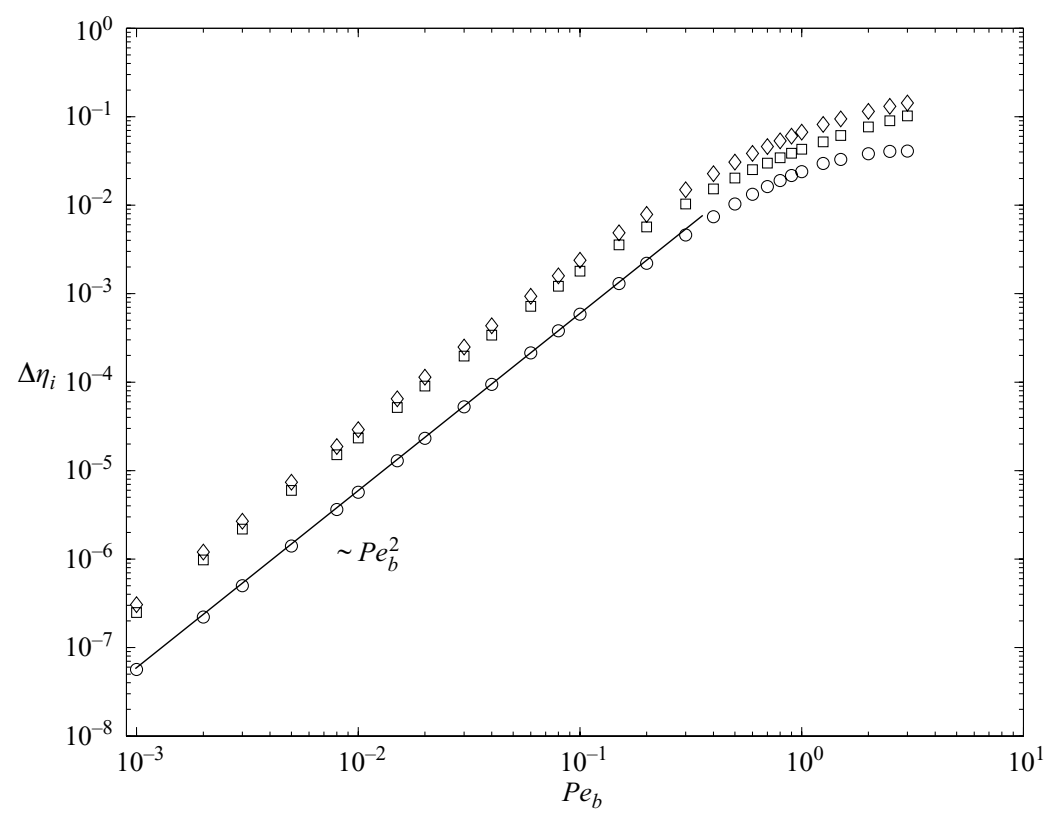

Figure 12. Small $P e_{b}$ variation of the intrinsic microviscosity for $\hat{b}=1.00001$. The following contributions are shown: $\bigcirc, \Delta \eta_{i}=2.084-\eta_{i} ; \square, \Delta \eta_{i}^{H}=\eta_{i}^{H}-1.828 ; \diamond, \Delta \eta_{i}^{B}=0.256-\eta_{i}^{B}$. The solid line indicates that the initial variation is proportional to $P e_{b}^{2}$.

and the Brownian contribution $\eta_{i}^{B}=0.256$. (For $\hat{b}=1.00001 \approx 1$ the interparticle-force plays essentially no role in determining the microrheology of the dispersion and $\eta_{i}^{P}$ is negligible as compared to the hydrodynamic and Brownian microviscosities.) To focus on the small $P e_{b}$ behaviour of the intrinsic microviscosity, we introduce the microviscosity variations: $\Delta \eta_{i}=2.084-\eta_{i}$ (total); $\Delta \eta_{i}^{H}=\eta_{i}^{H}-1.828$ (hydrodynamic); and $\Delta \eta_{i}^{B}=0.256-\eta_{i}^{B}$ (Brownian). The variations are defined to be positive and are plotted in figure 12 as a function of $P e_{b}$. The hydrodynamic variation is a monotonically increasing function of $P e_{b}$, as in the macroviscosity results of Bergenholtz et al. (2002). This fact is taken as further confirmation of the validity of our low $P e_{b}$ results in the context of applying the outer boundary at a finite distance $s_{f a r}$.

The increase in the Brownian microviscosity variation outweighs that of the hydrodynamic variation, resulting in a force-thinning intrinsic microviscosity at small $P e_{b}$. Figure 12 shows that the initial force-thinning is proportional to $P e_{b}^{2}$ : this scaling arises from a combination of the $O\left(P e_{b}^{2}\right)$ perturbation to the inner structural deformation, $P e_{b}^{2}\left(\hat{\boldsymbol{s}} \hat{\boldsymbol{s}}: \hat{\boldsymbol{F}}^{e x t} \hat{\boldsymbol{F}}^{e x t} f_{2}(s)+h_{2}(s)\right)$, which produces a hydrodynamic variation of $O\left(P e_{b}^{2}\right)$ and the $O\left(P e_{b}^{3}\right)$ perturbation to the inner structure, $P e_{b}^{3} f_{3}$, giving a Brownian variation of $O\left(P e_{b}^{2}\right)$. Although the analysis above has been restricted to $\hat{b}=1.00001$, we find the microviscosity force-thins proportional to $P e_{b}^{2}$ for all values of $\hat{b}$ studied.

We now turn to the microstructure at high $P e_{b}$. In figure 13 the contact value of the pair-distribution function is plotted versus the polar angle $\theta$ at several $P e_{b}$ for the case of particles with almost full hydrodynamic interactions, $\hat{b}=1.00001$. The boundarylayer structure is broadly similar to the case with no hydrodynamic interactions (cf. figure 10), with a large accumulation of pair density in the compressional region and a depletion of pair density in the extensional region. As $P e_{b}$ is increased, the 


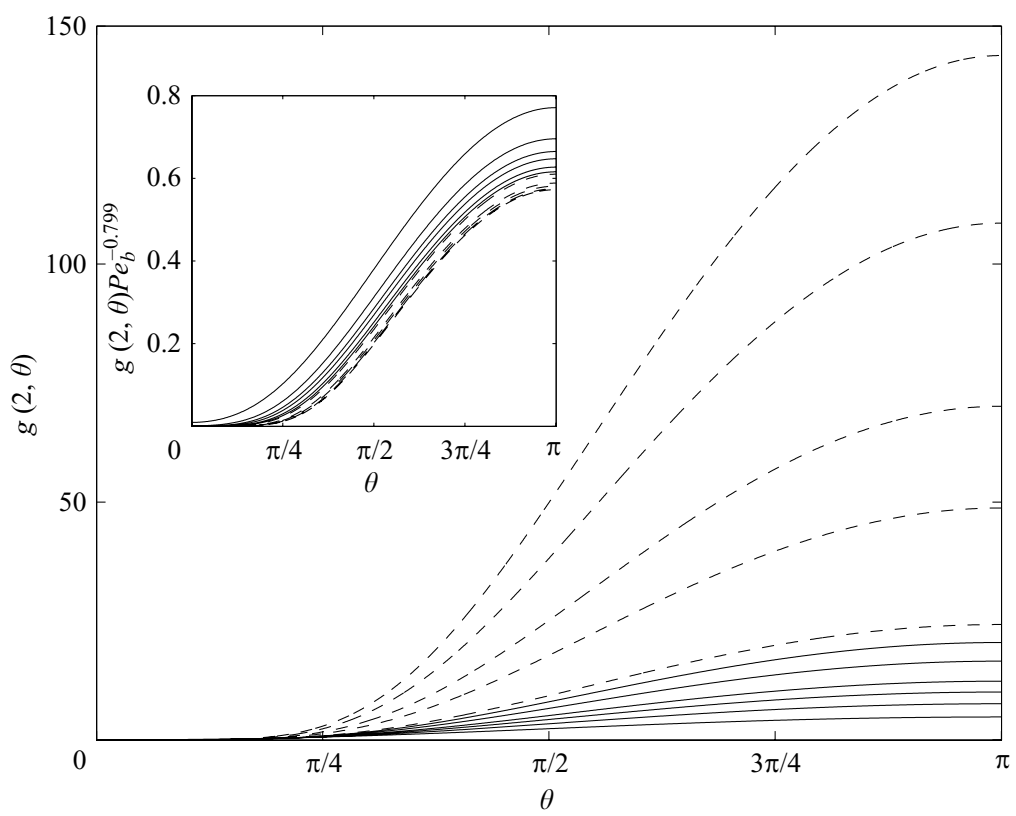

FIGURE 13. The pair-distribution function at contact as a function of the polar angle $\theta$ and $P e_{b}$ in the case of near-full hydrodynamic interactions $\hat{b}=1.00001$. Results from the solution of the full Smoluchowski equation are shown as solid lines whilst solutions from the boundary-layer equation are displayed as broken lines. From bottom-to-top: $P e_{b}=10,20,30,40,60,80$ and 100; and from the boundary-layer equation $P e_{b}=250,400,700,850$ and 1000. The inset displays the same data scaled by the theoretical prediction $g(2, \theta) \sim P e_{b}^{0.799}$ (see Appendix E), which is seen to collapse the data well.

accumulation and depletion become more pronounced; however, there are some subtle differences in the detailed boundary-layer characteristics. For a given value of $P e_{b}$ the contact value of the pair-distribution function in the compressional region is markedly smaller than for particles with no hydrodynamic interactions. This may be understood in terms of the hydrodynamic lubrication forces: in the compressional region the coming together of a force-free particle and the probe particle is hampered by the need to expel the solvent fluid from the narrow gap separating them. As is well known, as the gap becomes smaller, the force required to remove the remaining solvent diverges. This reduction in relative mobility at small interparticle separations (which is not present in the absence of hydrodynamic interactions) is responsible for the smaller accumulation of pair-density in the compressional region. In the extensional region, the pulling apart of a force-free particle from the probe by the advective velocity field is resisted by the flow of solvent into the increasing gap between the particles, which leads to an increase in the pair density in the extensional region.

Another important consequence of hydrodynamic interactions is that the contact value of the pair-distribution function does not scale linearly with $P e_{b}$, as was the case for $\hat{b} \rightarrow \infty$. In figure 14 , we plot $\ln \left(g_{\max }\right)$ (where $g_{\max }=g(2, \pi)$ is the maximum contact value of the pair-distribution function) versus $\ln \left(P e_{b}\right)$ and determine a sublinear scaling of $g(2) \sim P e_{b}^{0.773}$ for $\hat{b}=1.00001$. The exponent 0.773 may be predicted by a boundary-layer analysis of the Smoluchowski equation in the limit $P e_{b} \rightarrow \infty$ for the case $\hat{b} \equiv 1$. In Appendix E, it is shown that in this limit the pair-distribution function 


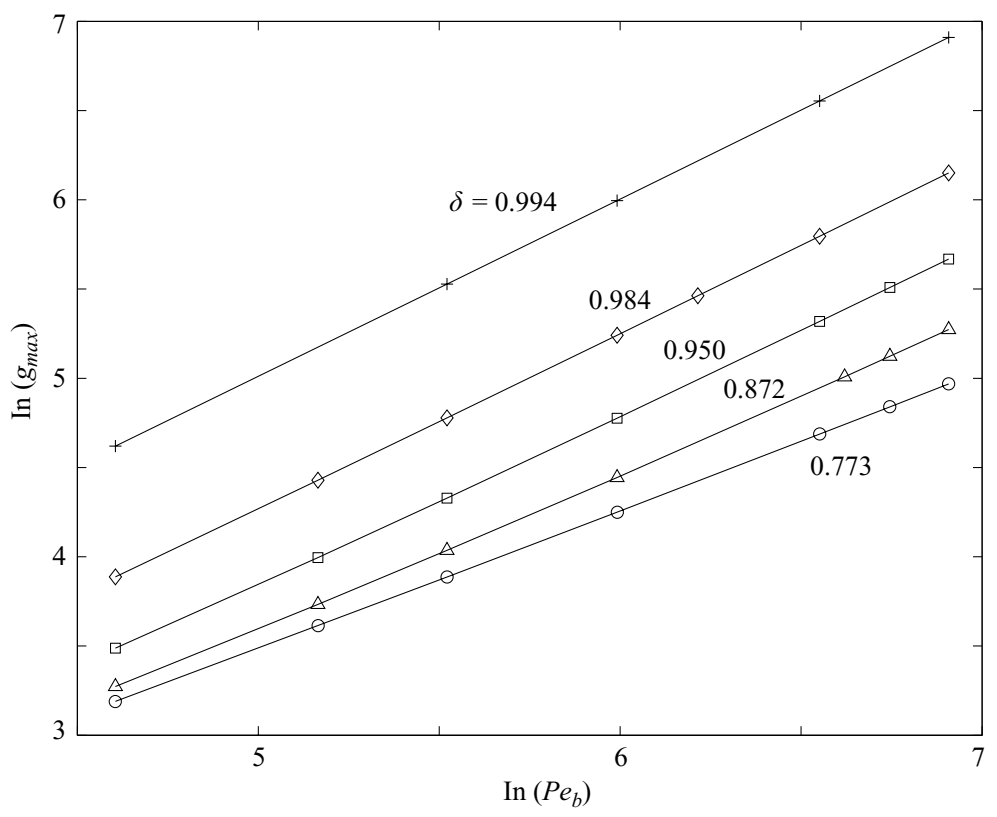

FIGURE 14. Determination of the scaling exponent $\delta$ relating the pair-distribution function at contact to $P e_{b}$ for various $\hat{b}: \bigcirc, \hat{b}=1.00001 ; \triangle, \hat{b}=1.001 ; \square, \hat{b}=1.01 ; \diamond, \hat{b}=1.1 ;+, \hat{b} \rightarrow \infty$. Here, $g_{\max }=g(2, \pi)$ denotes the maximum value of the pair-distribution function at contact.

in the boundary layer is given by

$$
g(s, \theta)=g_{0} \Gamma\left(\frac{H_{0}}{G_{1}}\right)\left(\frac{-\alpha P e_{b}}{Y(\theta)}\right)^{W_{0} / G_{1}} M\left(\frac{W_{0}}{G_{1}}, 1, \frac{\alpha P e_{b}(s-2)}{Y(\theta)}\right),
$$

where $H_{0}=0.402, G_{1}=2$ and $W_{0}=1.598$ are leading-order expansions of the hydrodynamic mobility functions $H(s), G(s)$, and $W(s)$ about $s=2$, respectively. In (6.3), $g_{0}$ is a constant which is determined by matching to the advective outer solution, $\alpha$ is a constant of arbitrary magnitude but of negative sign, $Y(\theta)$ represents the boundary-layer thickness (cf. (E 13)), $\Gamma$ is the gamma function, and $M$ is the first confluent hypergeometric function (Kummer's function). At contact $(s=2)$ we find $g(2, \theta) \sim P e_{b}^{W_{0} / G_{1}}=P e_{b}^{0.799}$, in good agreement with the numerically determined exponent of 0.773 from figure 14 . The small discrepancy between the two exponents may be explained by noting that the theoretical exponent is strictly only valid in the asymptotic limit $P e_{b} \rightarrow \infty$, whilst the numerical exponent is determined using contact value data in the range $100 \leqslant P e_{b} \leqslant 1000$. Nevertheless, as shown in the inset of figure 13 , the theoretical scaling prediction performs admirably in collapsing the numerical data.

The discussion presented above raises the question of how the scaling of the contact value of the pair-distribution function with $P e_{b}$ varies with $\hat{b}$. In a study of the microstructure of a sheared suspension at large $P e_{b}$, Brady \& Morris (1997) conclude, via an analytical boundary-layer study of the appropriate Smoluchowski equation, that $g(2) \sim P e_{b}$ for all $\hat{b}$ except in the so-called case of 'pure-hydrodynamics' $\hat{b} \equiv 1$, where $g(2) \sim P e_{b}^{0.78}$. Stokesian dynamics simulations of concentrated Brownian suspensions (at volume fractions of $\phi=0.30$ and $\phi=0.45$ ) in simple-shear flow performed by Morris \& Katyal (2002) indicate that $g(2) \sim P e_{b}^{0.70}$ for $b=1.00025$ in the range $1 \leqslant$ $P e_{b} \leqslant 1000$, as opposed to the linear scaling predicted by Brady \& Morris (1997). This 


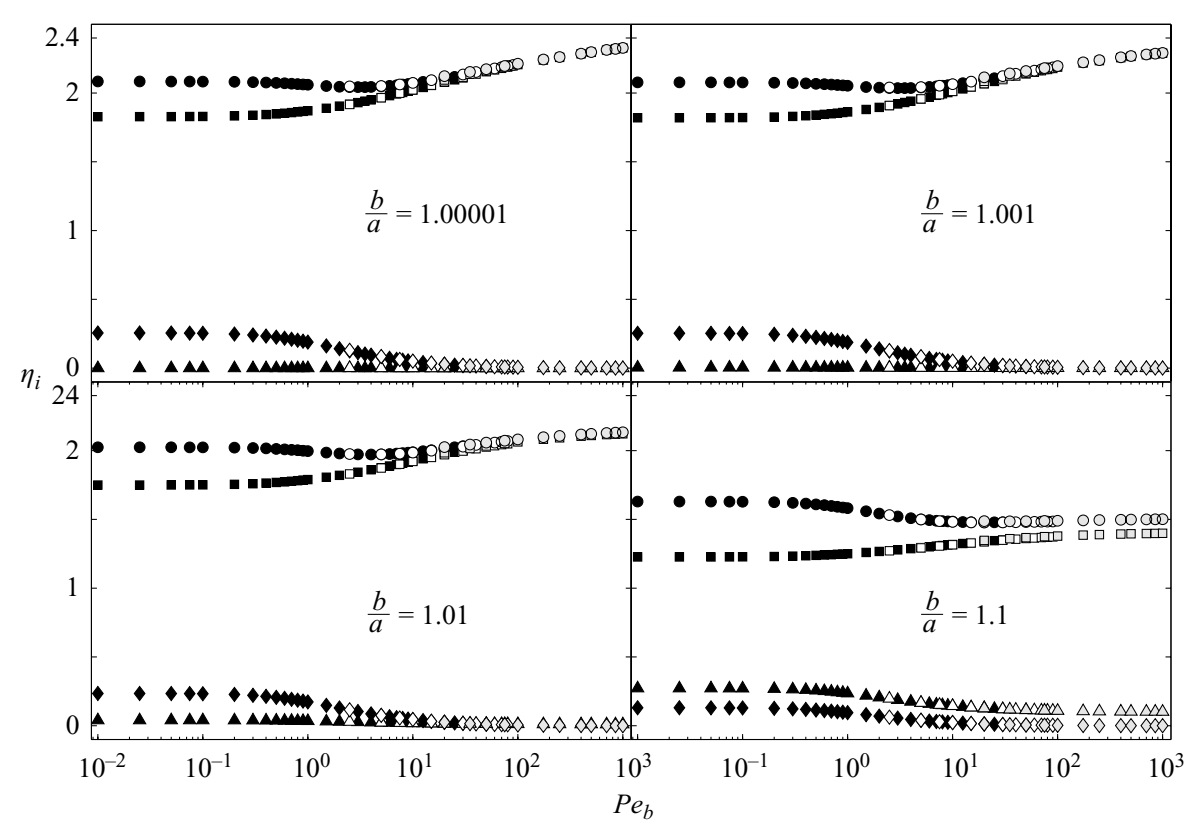

FIGURE 15. Contributions to the intrinsic microviscosity $\eta_{i}$ as a function of $P e_{b}$ for various $\hat{b}: \bigcirc$, total; $\square$, hydrodynamic; $\diamond$, Brownian; $\triangle$, interparticle force. Solid symbols, Legendre polynomial expansion; open symbols, finite-difference solution of full Smoluchowski equation; and grey symbols, finite-difference solution of the boundary-layer equation.

discrepancy in the two studies may be attributed to the neglect of the divergence of the relative-velocity field of two particles by Brady \& Morris (1997), which plays a crucial role in setting the microstructure at small interparticle separations for suspensions possessing very short-range excluded volume interactions $(\hat{b}-1 \ll 1)$. Mathematically, the non-zero divergence of the relative-velocity field states that the phase space of pair-trajectories is not volume conserving; physically, it acts as a source of pair-density in the compressional regions of the flow and a sink in the extensional regions. (Note, although the relative-velocity field of two particles has a non-zero divergence, the Newtonian suspending fluid is, of course, incompressible.) Let us define the exponent $\delta=\delta(\hat{b})$ such that $g(2) \sim P e_{b}^{\delta}$. In figure 14 , the exponent $\delta$ is determined for several values of $\hat{b}$ using data from our numerical solution of the Smoluchowski equation in the range $100 \leqslant P e_{b} \leqslant 1000$. We find that $\delta$ changes continuously between the limits of $\delta=0.773$ at $\hat{b}=1.00001$ to $\delta=1$ as $\hat{b} \rightarrow \infty$. Whether this continuous change may be predicted by an analytical boundary-layer theory of the Smoluchowski equation for arbitrary $\hat{b}$ is left as a future study.

In addition to affecting the microstructure of the dispersion, hydrodynamic interactions play a profound role in setting its microrheology. Let us first consider the microrheology of the suspension for $\hat{b}$ close to unity, where the thermodynamic radius $b$ is only slightly larger than the hydrodynamic radius $a$, so that the particles experience nearly full hydrodynamic interactions. In figure 15 , we plot the intrinsic microviscosity and its three constituents as a function of $P e_{b}$ for $\hat{b}=1.00001, \hat{b}=1.001, \hat{b}=1.01$ and $\hat{b}=1.1$. For $\hat{b}=1.00001$ and $\hat{b}=1.001$, it is observed that the interparticle contribution to the intrinsic microviscosity is essentially negligible as compared to the Brownian and hydrodynamic components. For small $P e_{b}$, the intrinsic viscosity exhibits a 


$\begin{array}{lllccc} & \eta_{i} & \eta_{i, 0}^{H} & \eta_{i, c} & \eta_{i, e} & \eta_{i, e} /\left(\eta_{i, e}+\eta_{i, c}\right) \\ \hat{b}=1.00001 & 2.3275 & 1.8280 & 0.3244 & 0.1751 & 0.351 \\ \hat{b} \rightarrow \infty & 1.0043 & 0 & 1.0032 & 0.0011 & 0.001\end{array}$

TABLE 1. Total $\eta_{i}$; equilibrium $\eta_{i, 0}^{H}$; compressional $\left(F_{r}^{\text {ext }}>0\right) \eta_{i, c}$; and extensional $\left(F_{r}^{\text {ext }}<0\right)$ $\eta_{i, e}$ contributions to the intrinsic microviscosity at $P e_{b}=1000$ for full hydrodynamics $(\hat{b}=1.00001 \approx 1)$ and without hydrodynamics $(\hat{b} \rightarrow \infty)$.

Newtonian plateau which is primarily determined by the hydrodynamic contribution, although a smaller, yet significant, Brownian contribution is present. Of course, the value of the intrinsic microviscosity at this plateau is merely the $O\left(\phi_{b}\right)$ correction to the long-time self-diffusivity, as discussed in $\S 4.2$. On increasing $P e_{b}$ to $O(1)$, the Brownian and hydrodynamic microviscosities decrease and increase, respectively. The decrease in the Brownian contribution outweighs the increase in the hydrodynamic contribution (cf. figure 12) causing the intrinsic microviscosity to force-thin up to $P e_{b} \approx 3$ for $\hat{b}=1.00001$ and $P e_{b} \approx 3.5$ for $\hat{b}=1.001$, at which point it attains a minimum. Beyond this minimum, the Brownian contribution becomes negligible and the hydrodynamic contribution increases steadily, which has the net effect of making the intrinsic microviscosity force-thicken. As $P e_{b}$ is increased, the advective flux (set up through the relative velocity field induced by the external force on the probe) of force-free particles towards the probe particle becomes stronger. For large $P e_{b}$, this advective flux 'squeezes' particles into close-contact with the probe, where they experience highly resistive lubrication forces, which is, in part, the cause of continuous force-thickening for $P e_{b} \sim O(1)$ and beyond. However, this is not the whole story: the moving probe not only 'pushes' background particles (leading to a high probabilitydensity in the boundary layer at the front of the probe), but also 'drags' background particles that are immediately behind it, as these particles are 'stuck' to the probe owing to the lubrication forces (cf. the higher probability-density downstream of the probe with hydrodynamics (figure 13) as compared to without hydrodynamics (figure 10)). Indeed, this 'dragging' effect contributes significantly to the microviscosity at large $P e_{b}$. In table 1 , we split the intrinsic microviscosity at $P e_{b}=1000$ into equilibrium $\eta_{i, 0}^{H}$, compressional (or pushing) $\eta_{i, c}$, and extensional (or dragging) $\eta_{i, e}$ contributions for full hydrodynamics $(\hat{b}=1.00001 \approx 1)$ and no hydrodynamics $(\hat{b} \rightarrow \infty)$. For full hydrodynamics $\eta_{i, e} /\left(\eta_{i, e}+\eta_{i, c}\right)=0.351$, whilst without hydrodynamics, for which there is no dragging effect (as the particles do not experience lubrication forces), $\eta_{i, e} /\left(\eta_{i, e}+\eta_{i, c}\right)=0.001$. The qualitative picture of the microviscosity as a function of $P e_{b}$ agrees with that of Bergenholtz et al. (2002) and the Stokesian dynamics simulations of concentrated sheared suspensions of Foss \& Brady (2000) for the macroviscosity.

On increasing $\hat{b}$, the qualitative description presented above changes; for $\hat{b}=1.1$ (again, see figure 15) the intrinsic microviscosity has significant contributions from all three of its constituents. The interparticle-force contribution is now greater than the Brownian contribution for all $P e_{b}$. Hydrodynamic interactions directly influence the Brownian microviscosity, (3.16), in the coupling of the diffusive motion of a pair of spheres. As the effect of hydrodynamic interactions diminishes with increasing $\hat{b}$ so does the Brownian microviscosity. (In the limit $\hat{b} \rightarrow \infty$, the diffusive motion of a pair of spheres is uncoupled, the relative diffusivity being simply a sum of their individual Stokes-Einstein-Sutherland diffusivities; consequently, the Brownian microviscosity 


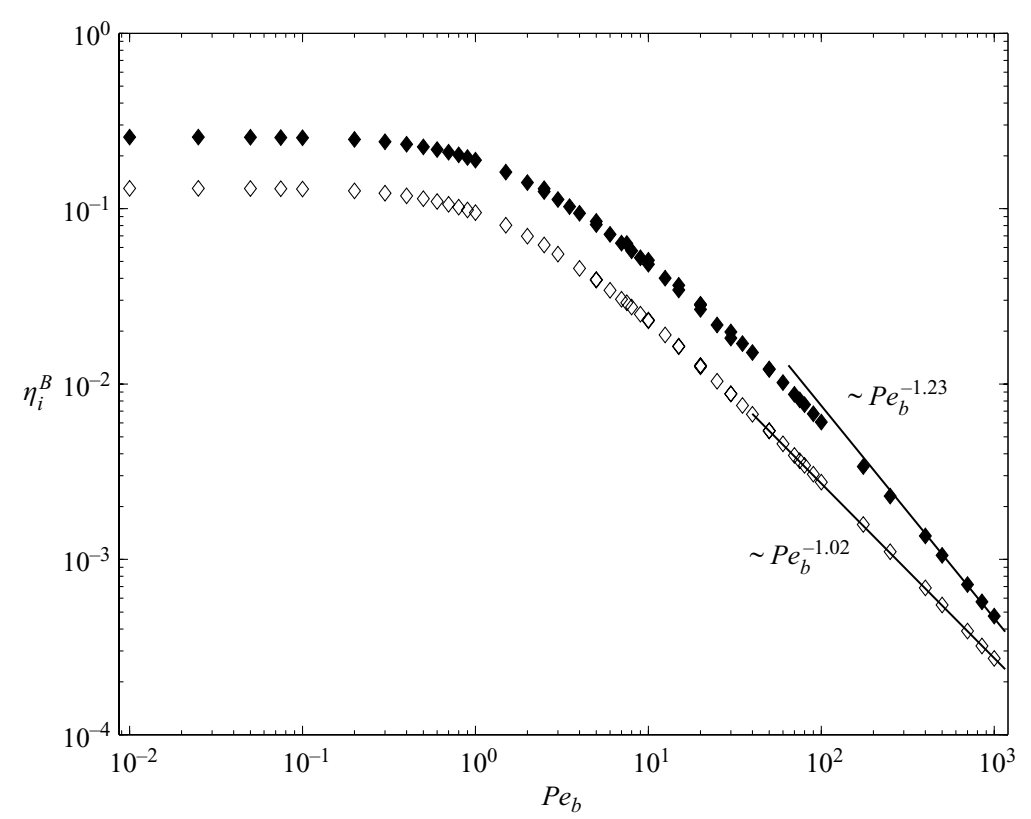

FIGURE 16. The Brownian intrinsic microviscosity $\eta_{i}^{B}$ as a function of $P e_{b}$ for $\hat{b}=1.00001$ (filled diamonds) and $\hat{b}=1.1$ (empty diamonds). The two solid lines are the high $P e_{b}$ scaling predictions $\eta_{i}^{B} \sim P e_{b}^{\delta-2}$ for each value of $\hat{b}$.

vanishes.) For $P e_{b} \ll 1$, the intrinsic microviscosity again attains a Newtonian plateau, whose major contribution is from the hydrodynamic microviscosity. As $P e_{b}$ is increased, the thinning of the interparticle and Brownian microviscosities is greater than the thickening of the hydrodynamic microviscosity, causing the intrinsic microviscosity to force-thin up to about $P e_{b} \approx 5$. Beyond this, the weak force-thickening of the hydrodynamic microviscosity is balanced by the force-thinning of the interparticle microviscosity (the Brownian microviscosity being essentially negligible for $P e_{b}>5$ ), resulting in a near-Newtonian high $P e_{b}$ plateau. The level of force-thickening of the hydrodynamic microviscosity is less than for the cases $\hat{b}=1.00001$ and $\hat{b}=1.001$ as the particles no longer experience the highly resistive lubrication forces when in close 'contact' (contact in a thermodynamic sense with respect to the excluded radius $b$ ).

Viewing $P e_{b}$ as the ratio of diffusion $\left(\tau_{D}\right)$ to advection $\left(\tau_{A}\right)$ time scales, the monotonic decay of the Brownian microviscosity with increasing $P e_{b}$ may be understood as follows: at low $P e_{b}$, we have $\tau_{D} / \tau_{A} \ll 1$, so that Brownian diffusion acts 'quickly' against the perturbation caused by the external force to restore a near-equilibrium microstructure throughout the suspension (up to distances of $O\left(P e_{b}^{-1}\right)$ ). However, on increasing $P e_{b}$, the equilibrating effect of Brownian diffusion is restricted to smaller distances and thus the Brownian microviscosity force-thins. For $P e_{b} \gg 1$, the action of Brownian diffusion is confined to an $O\left(P e_{b}^{-1}\right)$ thin boundary layer and the Brownian microviscosity follows an asymptotic force-thinning regime. From the expression (3.16) for $\eta_{i}^{B}$ and the scaling result $g(2) \sim P e_{b}^{\delta}$ at high $P e_{b}$, we see that the scaling of the Brownian microviscosity with $P e_{b}$ in the asymptotic force-thinning regime is $\eta_{i}^{B} \sim P e_{b}^{\delta-2}$. Figure 16 plots the Brownian microviscosity versus $P e_{b}$ for $\hat{b}=1.00001$ and $\hat{b}=1.1$. It is seen that the high $P e_{b}$ thinning regime is described well by the scaling prediction in both cases. Similarly, from the expression (3.15) 


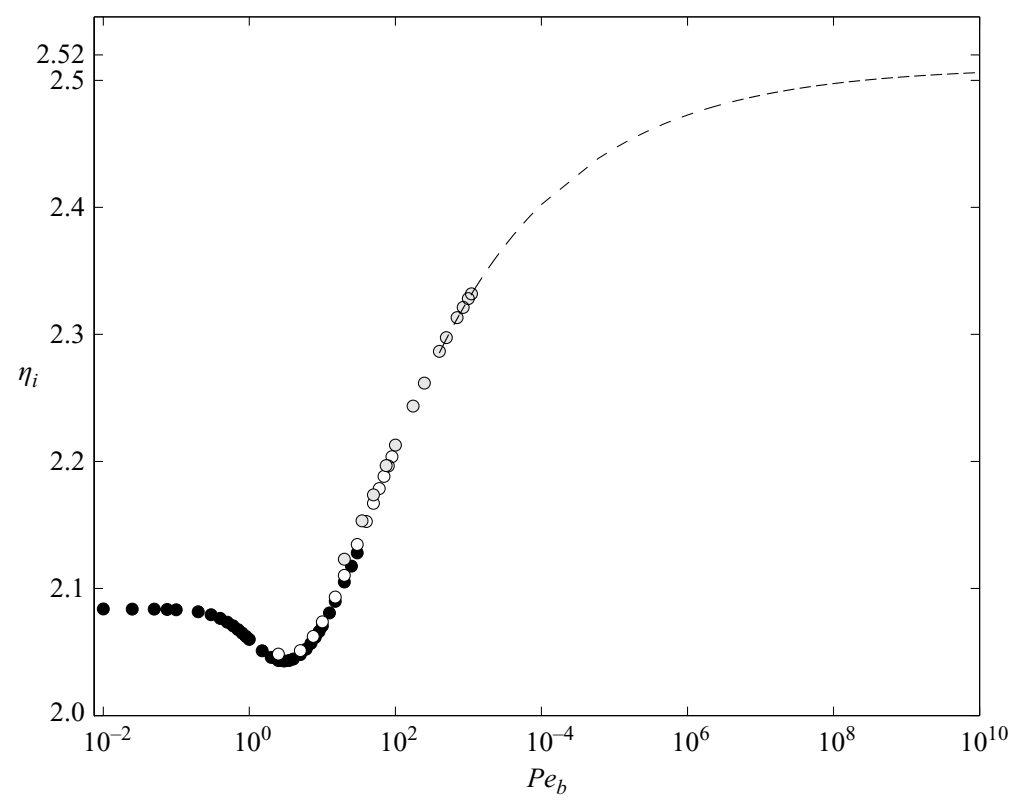

FIGURE 17. The intrinsic microviscosity for $\hat{b}=1.00001$ as a function of $P e_{b}$. The legend is the same as in figure 15 , with the addition of a dashed line indicating the extrapolation to infinite $P e_{b}: \eta_{i}^{H} \sim 2.511-0.879 P e_{b}^{-0.227}$.

for the interparticle microviscosity, we obtain the high $P e_{b}$ asymptotic behaviour $\eta_{i}^{P} \sim G(2 \hat{b}) P e_{b}^{\delta-1}$. This implies that for all finite $\hat{b}$ (for which $\delta<1$ ) the interparticle microviscosity should be a weakly decaying function $P e_{b}$ at large $P e_{b}$. Only in the absence of hydrodynamic interactions $(\hat{b} \rightarrow \infty$, where $\delta=1)$ does the interparticle microviscosity attain a true high $P e_{b}$ Newtonian plateau.

Unlike the Brownian and interparticle microviscosities, which for $P e_{b} \gg 1$ are determined primarily by the boundary-layer structure, the hydrodynamic microviscosity has contributions arising throughout the entire domain $s \geqslant 2$. The boundary layer has a characteristic size of $O\left(P e_{b}^{-1}\right)$ and contains an $O\left(P e_{b}^{\delta}\right)$ build-up of pair density, which from (3.13) implies an $O\left(P e_{b}^{\delta-1}\right)$ contribution to $\eta_{i}^{H}$. The $O(1)$ pair density in the advective 'outer' region $\left(s-2 \gg O\left(P e_{b}^{-1}\right)\right)$ yields an $O(1)$ (i.e. independent of $P e_{b}$ ) contribution to $\eta_{i}^{H}$. Thus, in the limit $P e_{b} \rightarrow \infty$, the hydrodynamic microviscosity takes the form $\eta_{i}^{H} \sim \alpha+\beta P e_{b}^{\delta-1}$, where $\alpha$ and $\beta$ are functions of $\hat{b}$ only. This asymptotic form may be used to extrapolate the large $P e_{b}$ results for $\eta_{i}^{H}$ to the $P e_{b} \rightarrow \infty$ limit. The coefficients $\alpha$ and $\beta$ are determined from a nonlinear regression analysis of $\eta_{i}^{H}$ versus $P e_{b}$ plots, using $\eta_{i}^{H}$ data for $P e_{b} \geqslant 200$. Figure 17 displays an extrapolation of the intrinsic microviscosity $\eta_{i}$ for $\hat{b}=1.00001$, for which $\eta_{i}^{H} \sim 2.511-0.879 P e_{b}^{-0.227}$ (recall that for $\hat{b}=1.00001$ at large $P e_{b}$, both $\eta_{i}^{B}$ and $\eta_{i}^{P}$ are negligible as compared to $\eta_{i}^{H}$ ). At infinite $P e_{b}$, the extrapolation predicts $\eta_{i}=2.511$, in good agreement with the studies of Batchelor \& Wen (1982) and Almog \& Brenner (1997) who both find that $\eta_{i}=2.52$ for $\hat{b} \equiv 1$ in the 'falling-ball' limit $P e_{b}^{-1} \equiv 0$. Furthermore, for $\hat{b} \equiv 1$ and $P e_{b}^{-1} \equiv 0$, the pair-distribution function is spherically symmetric (cf. (E 1$)$ ), i.e. for a given radial separation there is an equal probability of finding a background particle in the compressional or extensional regions around the probe. Hence, $\eta_{i}$ must have equal compressional/pushing $\left(\eta_{i, c}\right)$ and extensional/dragging $\left(\eta_{i, e}\right)$ contributions. 


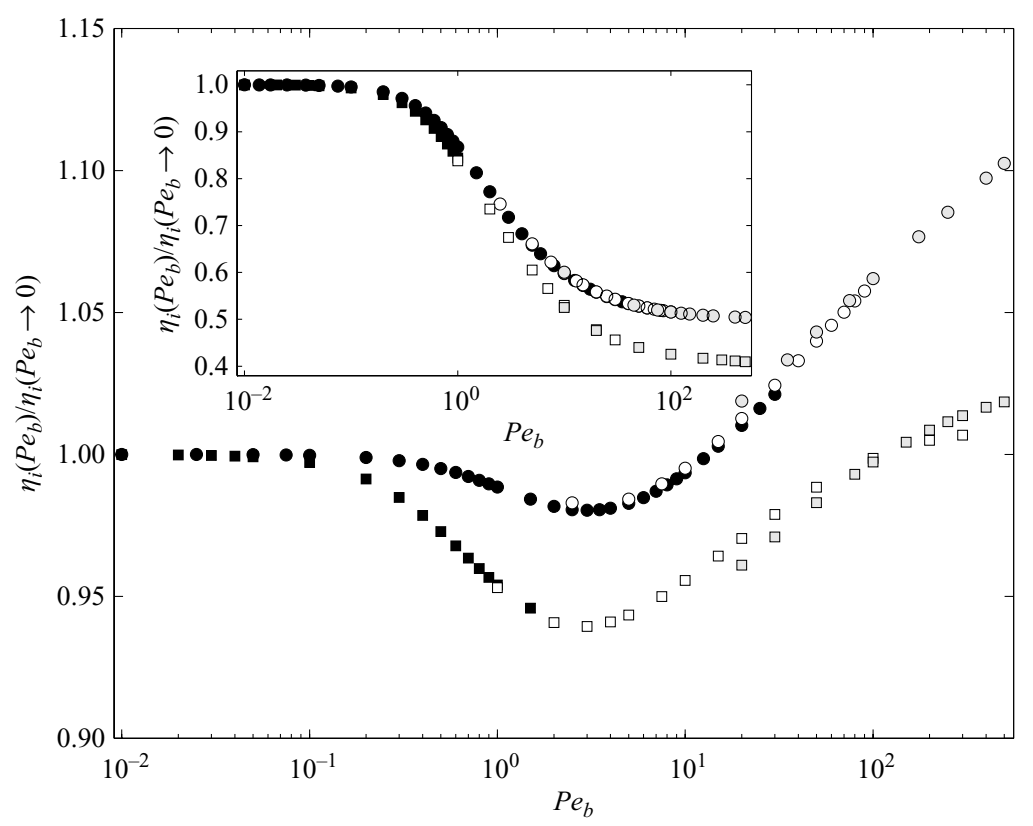

Figure 18. Comparison of the intrinsic microviscosity as a function of $P e_{b}$ for $\hat{b}=1.00001$ obtained in this investigation $(O)$ with the intrinsic macroviscosity results of Bergenholtz et al. (2002) ( $\square$ ). The filling pattern indicates the method of numerical solution as in figure 11. The inset shows a comparison of the intrinsic viscosities in the absence of hydrodynamic interactions $\hat{b} \rightarrow \infty$. For the microviscosity, the Péclet number is defined as $P e_{b}=F_{0} /(2 k T / b)$, whilst for the macroviscosity $P e_{b}=6 \pi \eta a b \dot{\gamma} /(2 k T / b)$, where $\dot{\gamma}$ is the shear-rate of the imposed shear flow.

As the equilibrium contribution $\eta_{i, 0}^{H}=1.83$ for $\hat{b} \equiv 1$ this gives $\eta_{i, c}=\eta_{i, e}=0.35$ at $P e_{b}^{-1} \equiv 0$. Now, at $P e_{b}=1000$ for $\hat{b}=1.00001 \approx 1$ (see table 1 ), we have $\eta_{i, c}=0.3244$ and $\eta_{i, e}=0.1751$, indicating that beyond $P e_{b}=1000$ force-thickening of the microviscosity is primarily due to the increasing accumulation of background particles in the extensional (downstream) region, until we attain a spherically symmetric microstructure in the limit $P e_{b} \rightarrow \infty$ (at which point $\eta_{i, c}=\eta_{i, e}$ ).

\section{Discussion}

The work presented in the previous sections attempts to offer a simple paradigm for active and nonlinear particle-tracking microrheology experiments. As a model system, we choose to study the motion of an externally forced Brownian probe particle in a dilute colloidal dispersion of force- and torque-free particles. In particular, it has been shown how the average translational velocity of the probe may be used to define the microviscosity of the dispersion via application of Stokes drag law. Whilst being simple enough to allow analytical and numerical treatment, the model nevertheless exhibits non-trivial rheological properties. It is of interest to contrast the results of this investigation to those obtained from a conventional macrorheological study, such as performed by Bergenholtz et al. (2002), who examined the microstructure and macrorheology of a dilute colloidal dispersion in an ambient shear flow. In figure 18, we compare the intrinsic microviscosity obtained in this work with the intrinsic macroviscosity computed by Bergenholtz et al. (2002) as a function of $P e_{b}$ for $\hat{b}=1.00001$. 
The intrinsic macroviscosity $\eta_{i}$ is defined as the $O\left(\phi_{b}^{2}\right)$ coefficient of the relative macroviscosity: $\eta_{r}=1+5 \phi / 2+\eta_{i} \phi_{b}^{2}$ (the $O(\phi)$ term of the relative macroviscosity is, of course, the single-particle Einstein correction, which scales with the hydrodynamic radius $a$ ), whereas the intrinsic microviscosity is defined as the $O\left(\phi_{b}\right)$ coefficient of the relative microviscosity (cf. (3.12)). To aid in the comparison, the intrinsic viscosity for both sets of data is normalized by its limiting value as $P e_{b} \rightarrow 0$, which in our case is the $O\left(\phi_{b}\right)$ correction to the long-time self-diffusivity and for the macrorheology problem is the low-frequency dynamic, or steady shear, macroviscosity. The general functional behaviour of the intrinsic viscosity is seen to be similar for the two cases. In the limit $P e_{b} \rightarrow 0$, the intrinsic viscosity exhibits a Newtonian low-force(shear) plateau, which on increasing $P e_{b}$ is succeeded by a force(shear)-thinning regime, caused by a decreasing Brownian contribution that outweighs the increasing hydrodynamic contribution. This force(shear)-thinning is initially proportional to $P e_{b}^{2}$ and persists until $P e_{b} \sim O(1)$, where the intrinsic viscosity attains a minimum value. The minimum occurs at $P e_{b} \approx 3$ for both the microviscosity and macroviscosity. Beyond this minimum, the Brownian contribution is negligible and the intrinsic viscosity is determined primarily by the hydrodynamic contribution. Since the hydrodynamic viscosity is a monotonically increasing function of $P e_{b}$, the viscosity force(shear)-thickens: the degree of thickening is significantly greater for the microviscosity $\left(\eta_{i} / \eta_{i, 0} \approx 1.10\right.$ at $\left.P e_{b}=500\right)$ as compared to the macroviscosity $\left(\eta_{i} / \eta_{i, 0} \approx 1.02\right.$ at $\left.P e_{b}=500\right)$. Our results support the claim of Bergenholtz et al. (2002) that shear-thickening of the macroviscosity of colloidal dispersions at high $\mathrm{Pe}_{b}$ is a two-body (or dilute) effect, which arises as a consequence of the boundary-layer formation at small interparticle separations.

When the particles do not experience hydrodynamic interactions $(\hat{b} \rightarrow \infty)$, the intrinsic microviscosity and intrinsic macroviscosity are determined exclusively from the interparticle-force contribution. It was found that the microviscosity thins monotonically with increasing $P e_{b}$ from a Newtonian plateau in the limit $P e_{b} \rightarrow 0$ to a second Newtonian plateau at infinite $P e_{b}$. Qualitatively similar behaviour is seen for the intrinsic macroviscosity, as shown by the inset of figure 18 .

The comparison of micro- and macro-viscosity raises several issues that warrant further elaboration. As noted above, the micro- and macro-viscosity scale differently with the background particle volume fraction $\phi_{b}$ : the microstructurally-dependent contribution (which has hitherto been termed the intrinsic viscosity) to the microviscosity is $O\left(\phi_{b}\right)$, whilst for the macroviscosity it is $O\left(\phi_{b}^{2}\right)$. Knowing these scalings in advance allows us to compare the micro- and macro-viscosity in a consistent manner. However, for more complicated (or unknown) materials, such scalings may not be known a priori, and the comparison between micro and macro may not be so agreeable. Nonetheless, as mentioned in $\S 1$, disparities between microand macro-rheological measurements highlight the fundamental physical differences in the two techniques. Therefore, such discrepancies do not render the microrheological data invalid; on the contrary, we should strive to understand the additional information contained in them, and, to this end, it is essential to develop detailed theoretical models for active microrheology experiments.

The comparison in figure 18 is between the microviscosity at fixed force and the macroviscosity at fixed shear rate. Properly, we should compare the fixed-force microviscosity to the fixed-stress macroviscosity (and, likewise, fixed-velocity micro to fixedshear rate macro). However, in the dilute limit, it is easy to show that the fixed-stress and fixed-shear rate macroviscosities are identical. Thus, the comparison in figure 18 is legitimate. In contrast, as demonstrated by Squires \& Brady (2005) and discussed below, the fixed-force and fixed-velocity microviscosities are different in the dilute limit. 
Furthermore, in the fixed-velocity mode, the probe does not move diffusively, and the relative diffusivity is the background particle (Stokes-Einstein-Sutherland) diffusivity $D$. In fixed-force mode, the probe moves deterministically and diffusively; thus, the relative diffusivity is $2 D$. Therefore, the Péclet number for fixed-velocity $\left(F_{0} /(k T / b)\right)$ is twice that for fixed force $\left(F_{0} /(2 k T / b)\right)$. Lastly, although we can compare the microand macro-viscosity, microrheology can only determine a scalar viscosity (at least for a single spherical probe; a non-spherical probe or two spherical probes may yield more information), whereas in macrorheology the full stress tensor is obtainable, including normal stress differences and an isotropic osmotic pressure (Bergenholtz et al. 2002).

In this study it was assumed (for simplicity) that the probe particle is of the same size as the background particles; in practice, this is not always the case. For instance, Habdas et al. (2004) in their investigation of the forced motion of a magnetic bead in a dense colloidal dispersion used bead particles of roughly twice the size of the dispersion particles. A natural question to pose is: how does the calculated microviscosity of the dispersion vary with the size of the probe particle? Almog \& Brenner (1997) have addressed this question in the so-called 'falling-ball' limit, $P e^{-1} \equiv 0$, for $\hat{b} \equiv 1$. They find that when the probe sphere is much larger than the suspended spheres one recovers Einstein's viscosity correction $\eta_{i}=2.5$. On the other hand, when the probe is much smaller than the suspended spheres they observe $\eta_{i} \rightarrow \infty$. In the absence of hydrodynamic interactions $(\hat{b} \rightarrow \infty)$, Squires \& Brady (2005) have solved the pair problem for all $P e_{b}$ and all size ratios. They show that $\eta_{i} \sim(1+\lambda)^{2} / 2$, where $\lambda$ is the size ratio of probe particle to background particle. It would be of interest to examine how the microviscosity varies with the size of the probe particle at finite $P e_{b}$ and whether or not the scaling results of Squires \& Brady (2005) hold as hydrodynamic interactions are brought in.

An alternative to fixing the force on the probe is to fix its velocity. In this case, the ensemble-averaged force on the probe may now be related to the suspension's microviscosity via the Stokes drag formula. A natural question arises as to whether the fixed-force and fixed-velocity procedures give the same intrinsic microviscosity. Almog \& Brenner (1997) have shown in the 'falling-ball' limit $\left(P e_{b} \rightarrow \infty\right)$ that the intrinsic microviscosity does differ when calculated using fixed-force or fixed-velocity probe particles. In fact, the intrinsic viscosity measured using the fixed-velocity probe is always greater than that obtained for the fixed-force probe, except in the limit where the probe particle is much larger than the suspended spheres for which one recovers Einstein's viscosity correction in both cases. In the absence of hydrodynamic interactions and for all $P e_{b}$, Squires \& Brady (2005) find that the ratio of fixedvelocity to fixed-force intrinsic microviscosities is $(1+\lambda) / \lambda$. Once again, the fixedvelocity microviscosity is always greater than the fixed-force microviscosity except in the limit where the probe is much larger than the background particles, where they are equal. Physically speaking, the fixed-velocity probe expends more energy in pushing surrounding particles out of its path than the fixed-force probe which may pass around any obstructing particles. Almog \& Brenner (1997) state the discrepancy between the fixed-force and fixed-velocity microviscosities to be indicative of the fundamentally non-continuum nature of the suspension. Whether this discrepancy persists for finite $P e_{b}$ is not known (except in the special case $\hat{b} \rightarrow \infty$, Squires \& Brady 2005) and is clearly a question of interest as it suggests the intriguing possibility of applying microrheological techniques to studying the non-continuum nature of soft heterogeneous materials.

In conclusion, we may view this investigation as a step towards laying a theoretical foundation for active and nonlinear microrheology. Adopting the forced motion of 
a single-probe particle in an otherwise quiescent colloidal dispersion as a simple paradigm for active tracking experiments, we have shown that, when appropriately scaled, the microviscosity of the dispersion is in qualitative agreement with the macroviscosity. However, for more complex materials, where the relevant micro and macro scalings are not known a priori, micro- and macro-rheological data may not be in such good agreement, and great care is to be taken in the interpretation of the microrheology experiments. Nevertheless, our study suggests that active microrheology has the potential to be a valuable tool with which to explore the rich nonlinear rheology of complex fluids.

The authors wish to thank Dr Johan Bergenholtz for assistance in the finitedifference solution of the Smoluchowski equation and for supplying the macroviscosity data used in figure 18. We also thank Dr Helen Wilson for fruitful discussions regarding Appendix D. The work was supported in part by grant CTS - 050070 from the National Science Foundation.

\section{Appendix A. The Brownian velocity contribution}

In this Appendix the result (3.10) for the Brownian velocity contribution is derived. We consider a collection of colloidal particles subjected to a stochastic Brownian force $\boldsymbol{F}^{B}$. In what follows there is an implicit summation over all particles in the suspension.

The Brownian force is characterized by the usual statistical properties

$$
\overline{\boldsymbol{F}^{B}\left(t^{\prime}\right)}=0, \quad \overline{\boldsymbol{F}^{B}\left(t^{\prime}\right) \boldsymbol{F}^{B}\left(t^{\prime \prime}\right)}=\mathscr{F} \delta\left(t^{\prime}-t^{\prime \prime}\right),
$$

where the overbar denotes an average over the many collisions of the solvent molecules with the suspended particles. The appropriate time scale for this average is $t_{s}=$ $m_{s} / 6 \pi \eta a_{s}$ (where $m_{s}$ and $a_{s}$ are the mass and radius of a solvent molecule, respectively), i.e. the vorticity diffusion, or inertial relaxation, time of a solvent molecule. The amplitude of correlation of the Brownian force at times $t^{\prime}$ and $t^{\prime \prime}$ is given by the fluctuation-dissipation theorem as $\mathscr{F}=2 k T \boldsymbol{R}^{F U}$, with $\boldsymbol{R}^{F U}$ the multiparticle resistance tensor relating the forces on the particles to their velocities. This resistance tensor is a function of the instantaneous configuration, say $\boldsymbol{X}(t)$, of all the particles.

From low-Reynolds-number fluid dynamics, the instantaneous velocity of the particles owing to the Brownian force is $\boldsymbol{U}^{B}=\boldsymbol{M}^{U F} \cdot \boldsymbol{F}^{B}$, where $\boldsymbol{M}^{U F}=\left(\boldsymbol{R}^{F U}\right)^{-1}$ is the multiparticle mobility tensor that relates the velocities of the particles to the forces acting on them. We must average this velocity over a time step $\Delta t$, which is long compared to the inertial relaxation time of a particle, $t_{I}=m / 6 \pi \eta a$, but much smaller than the diffusive time scale, $t_{D}=a^{2} / D$, characterizing changes in the configuration of the particles. Doing so we find

$$
\begin{aligned}
\frac{1}{\Delta t} \int_{t}^{t+\Delta t} U_{i}^{B} \mathrm{~d} t^{\prime} & =\frac{1}{\Delta t} \int_{t}^{t+\Delta t} M_{i j}^{U F}\left(t^{\prime}\right) F_{j}\left(t^{\prime}\right) \mathrm{d} t^{\prime} \\
& =M_{i j}^{U F}(t) \frac{1}{\Delta t} \int_{t}^{t+\Delta t} F_{j}\left(t^{\prime}\right) \mathrm{d} t^{\prime}+\frac{\partial M_{i j}^{U F}(t)}{\partial x_{k}} \frac{1}{\Delta t} \int_{t}^{t+\Delta t} \Delta X_{k}\left(t^{\prime}\right) F_{j}\left(t^{\prime}\right) \mathrm{d} t^{\prime},
\end{aligned}
$$

where the summation convention is applied to repeated indices, and the configuration displacement is to leading order $\Delta X_{k}\left(t^{\prime}\right)=M_{k l}^{U F}(t) \int_{0}^{t^{\prime}} F_{l}\left(t^{\prime \prime}\right) \mathrm{d} t^{\prime \prime}$. Averaging (A 2) over 
many solvent collisions and using (A 1) we obtain

$$
\overline{\boldsymbol{U}^{B}}=k T \nabla \cdot \boldsymbol{M}^{U F},
$$

where the divergence is taken with respect to the last index of the mobility tensor. The velocity of an individual particle $\alpha$ is given by $\overline{\boldsymbol{U}_{\alpha}^{B}}=k T \sum_{\beta=1}^{N} \nabla_{\beta} \cdot \boldsymbol{M}_{\alpha \beta}^{U F}$, where the index $\beta$ runs over all $N$ particles. In the case of just two particles (say 1 and 2) we have $\overline{\boldsymbol{U}_{1}^{B}}=k T\left(\nabla_{1} \cdot \boldsymbol{M}_{11}^{U F}+\nabla_{2} \cdot \boldsymbol{M}_{12}^{U F}\right)$. Employing a centre-of-mass coordinate system $\left(\nabla_{r}=\nabla_{2}=-\nabla_{1}\right)$, the result (3.10) is recovered.

\section{Appendix B. Finite-difference method}

In this Appendix, we describe in detail the finite-difference solution of the Smoluchowski equation that was outlined in $\S 5.2$. The finite-difference solution is used for $P e_{b} \geqslant 2$, for which we expect a boundary layer adjacent to the probe in the compressional region of the relative-velocity field in which there are large gradients in the pair-distribution function. To capture accurately the behaviour of the pairdistribution function in the boundary layer, we stretch the radial coordinate via the transformation $y=P e_{b}(s-2)$. Thus, the Smoluchowski equation (5.1) becomes (with $\left.\hat{\boldsymbol{F}}^{e x t}=-\hat{z}\right)$

$$
\begin{aligned}
G \cos \theta \frac{\partial f}{\partial y} & -\frac{P e_{b}^{-1} H \sin \theta}{2 x} \frac{\partial f}{\partial \theta}+P e_{b}^{-1} W \cos \theta(1+f) \\
& =G \frac{\partial^{2} f}{\partial y^{2}}+P e_{b}^{-1}\left(\frac{\mathrm{d} G}{\mathrm{~d} s}+\frac{G}{x}\right) \frac{\partial f}{\partial y}+\frac{P e_{b}^{-2} H}{4 x^{2} \sin \theta} \frac{\partial}{\partial \theta}\left(\sin \theta \frac{\partial f}{\partial \theta}\right),
\end{aligned}
$$

where $x=1+P e_{b}^{-1} y / 2$. Whilst paying careful attention to the boundary-layer structure, it is also important to describe correctly the far-field behaviour of $f(s)$. To this end, we perform a second radial coordinate transformation to go from the semi-infinite domain $y \in[0, \infty)$ to the finite domain $t \in[0,1]$ via the mapping

$$
t=\exp \left(-\left\{\omega+\frac{1-\omega}{1+y}\right\} y\right)
$$

where $\omega$ is an adjustable parameter. The Smoluchowski equation now reads

$$
\begin{aligned}
G \cos \theta \frac{\mathrm{d} t}{\mathrm{~d} y} \frac{\partial f}{\partial t}- & \frac{P e_{b}^{-1} H \sin \theta}{2 x} \frac{\partial f}{\partial \theta}+P e_{b}^{-1} W \cos \theta(1+f) \\
= & G\left(\frac{\mathrm{d} t}{\mathrm{~d} y}\right)^{2} \frac{\partial^{2} f}{\partial t^{2}}+\left(G \frac{\mathrm{d}^{2} t}{\mathrm{~d} y^{2}}+P e_{b}^{-1}\left\{\frac{\mathrm{d} G}{\mathrm{~d} s}+\frac{G}{x}\right\} \frac{\mathrm{d} t}{\mathrm{~d} y}\right) \frac{\partial f}{\partial t} \\
& +\frac{P e_{b}^{-2} H}{4 x^{2} \sin \theta} \frac{\partial}{\partial \theta}\left(\sin \theta \frac{\partial f}{\partial \theta}\right),
\end{aligned}
$$

with the boundary conditions

$$
\begin{array}{rll}
\left(\frac{\mathrm{d} t}{\mathrm{~d} y}\right) \frac{\partial f}{\partial t}=\cos \theta(1+f) & \text { at } & t=1, \\
f=0 & \text { at } & t=0 .
\end{array}
$$


The Smoluchowski equation is discretized by approximating the radial and angular derivatives by central differences

$$
\begin{array}{ll}
\frac{\partial f}{\partial t}=\frac{f_{j+1, k}-f_{j-1, k}}{2 \Delta t}, & \frac{\partial^{2} f}{\partial t^{2}}=\frac{f_{j+1, k}-2 f_{j, k}+f_{j-1, k}}{\Delta t^{2}}, \\
\frac{\partial f}{\partial \theta}=\frac{f_{j, k+1}-f_{j, k-1}}{2 \Delta \theta}, & \frac{\partial^{2} f}{\partial \theta^{2}}=\frac{f_{j, k+1}-2 f_{j, k}+f_{j, k-1}}{\Delta \theta^{2}},
\end{array}
$$

where $j=1,2, \ldots, J$ and $k=0,1, \ldots, K$. The interior domain of solution is $0<t<1$ with $t=j /(J+1)$ (the points $t=0$ and $t=1$ are excluded as the radial boundary conditions must be applied there) and $0 \leqslant \theta \leqslant \pi$ with $\theta=k \pi / K$. Thus, the node spacings are $\Delta \theta=\pi / K$ and $\Delta t=1 /(J+1)$. Note that although the node spacing is constant in $t$-space, it is not in $s$-space, and via the adjustment of $\omega$ we can place a large number of nodes near $s=2$ to model the boundary layer accurately. We can make a vector comprising the unknown structural deformation at each grid point, $f_{j, k}$, by defining an index $i=j+k J$ running from 1 to $J(K+1)$. This enables the discretized Smoluchowski equation to be written as the matrix equation $\boldsymbol{A} \cdot \boldsymbol{f}=\boldsymbol{w}$, with $\boldsymbol{f}$ the unknown structural deformation vector. The coefficient matrix $\boldsymbol{A}$ is tridiagonal with two fringes, so we may write $\boldsymbol{T} \cdot \boldsymbol{f}=\boldsymbol{w}-\left(\boldsymbol{F}_{1}+\boldsymbol{F}_{2}\right) \cdot \boldsymbol{f}$, where $\boldsymbol{T}$ is the tridiagonal part of $\boldsymbol{A}$, and $\boldsymbol{F}_{1}$ and $\boldsymbol{F}_{2}$ are the fringes. (Here, the term fringe is used to denote a matrix whose tridiagonal elements are zero.) The matrix equation is solved by constructing the Jacobi iteration

$$
\boldsymbol{f}^{(n+1)}=\boldsymbol{T}^{-1} \cdot\left(\boldsymbol{w}-\left(\boldsymbol{F}_{1}+\boldsymbol{F}_{2}\right) \cdot \boldsymbol{f}^{(n)}\right),
$$

where $n$ is the iteration number. For a particular value of $P e_{b}\left(P e_{b, 2}\right.$ say) the iteration input $f_{P e_{b, 2}}^{(0)}$ is the converged result $f_{P e_{b, 1}}$ for the structural deformation at $P e_{b, 1}<P e_{b, 2}$, i.e. a parameter continuation in $P e_{b}$ is performed. The iteration is deemed to have converged if the norm $\left\|\boldsymbol{f}^{(n+1)}-\boldsymbol{f}^{(n)}\right\|<\epsilon$, where the tolerance $\epsilon$ is typically $10^{-14}$. Alternatively, the matrix equation may be written in component form as

$$
a_{i} f_{i-1}+b_{i} f_{i}+c_{i} f_{i+1}+d_{i} f_{i-J}+e_{i} f_{i+J}=w_{i},
$$

where the vectors constituting the tridiagonal matrix $\boldsymbol{T}$ are

$$
\begin{aligned}
& a_{i}=G\left(\frac{\mathrm{d} t}{\mathrm{~d} y}\right)^{2} \Delta t^{-2}-\frac{1}{2}\left(G \frac{\mathrm{d}^{2} t}{\mathrm{~d} y^{2}}+P e_{b}^{-1}\left\{\frac{\mathrm{d} G}{\mathrm{~d} s}+\frac{G}{x}\right\} \frac{\mathrm{d} t}{\mathrm{~d} y}\right) \Delta t^{-1}+\frac{G}{2} \cos \theta\left(\frac{\mathrm{d} t}{\mathrm{~d} y}\right) \Delta t^{-1}, \\
& b_{i}=-2 G\left(\frac{\mathrm{d} t}{\mathrm{~d} y}\right)^{2} \Delta t^{-2}-\frac{P e_{b}^{-2} H}{2 x^{2}} \Delta \theta^{-2}-P e_{b}^{-1} W \cos \theta, \\
& c_{i}=G\left(\frac{\mathrm{d} t}{\mathrm{~d} y}\right)^{2} \Delta t^{-2}+\frac{1}{2}\left(G \frac{\mathrm{d}^{2} t}{\mathrm{~d} y^{2}}+P e_{b}^{-1}\left\{\frac{\mathrm{d} G}{\mathrm{~d} s}+\frac{G}{x}\right\} \frac{\mathrm{d} t}{\mathrm{~d} y}\right) \Delta t^{-1}-\frac{G}{2} \cos \theta\left(\frac{\mathrm{d} t}{\mathrm{~d} y}\right) \Delta t^{-1},
\end{aligned}
$$

the fringe vectors are

$$
\begin{aligned}
& d_{i}=-\frac{P e_{b}^{-1} H \sin \theta}{4 x} \Delta \theta^{-1}+\frac{P e_{b}^{-2} H}{4 x^{2}} \Delta \theta^{-2}-\frac{P e_{b}^{-2} H}{8 x^{2}} \cot \theta \Delta \theta^{-1}, \\
& e_{i}=\frac{P e_{b}^{-1} H \sin \theta}{4 x} \Delta \theta^{-1}+\frac{P e_{b}^{-2} H}{4 x^{2}} \Delta \theta^{-2}+\frac{P e_{b}^{-2} H}{8 x^{2}} \cot \theta \Delta \theta^{-1},
\end{aligned}
$$


and the forcing vector is simply $w_{i}=P e_{b}^{-1} W \cos \theta$. It appears from the above that $d_{i}$ and $e_{i}$ are infinite at $\theta=0$ and $\theta=\pi$; however, this is just a coordinate singularity and can easily be eliminated by imposing a natural symmetry boundary condition $\partial f / \partial \theta=0$ (in finite-difference terms $f_{j, k+1}=f_{j, k-1}$ ) at $\theta=0$ and $\theta=\pi$.

\section{Appendix C. Boundary-layer equation}

Our point of departure is the Smoluchowski equation in spherical polar coordinates (5.1). To focus on the details of the boundary layer in the high $P e_{b}$ limit, we introduce the stretched radial coordinate $y=P e_{b}(s-2)$, yielding the following equation

$$
G \frac{\partial^{2} f}{\partial y^{2}}+Q \frac{\partial f}{\partial y}+\frac{P e_{b}^{-2} H}{4 \sin \theta} \frac{\partial}{\partial \theta}\left(\sin \theta \frac{\partial f}{\partial \theta}\right)=-P e_{b}^{-1}\left(\frac{H}{2} F_{\theta}^{e x t} \frac{\partial f}{\partial \theta}+W F_{r}^{e x t}(1+f)\right),
$$

where terms of $O\left(\mathrm{Pe}_{b}^{-3}\right)$ and higher have been neglected. The smallest term discarded is $O\left(y^{2} P e_{b}^{-3}\right)$ which indicates that (C1) is not uniformly valid. However, the loss of uniformity occurs at $y \sim O\left(P e_{b}^{1 / 2}\right)$ and hence should not affect the boundary-layer structure (for which $y \sim O(1)$ ) of the pair-distribution function at large $P e_{b}$. The function $Q$ is given by

$$
Q=F_{r}^{e x t} G+\frac{\mathrm{d} G}{\mathrm{~d} y}+P e_{b}^{-1} G\left(1-\frac{P e_{b}^{-1} y}{2}\right) .
$$

The boundary conditions on $f(y, \theta)$ are

$$
\begin{array}{rll}
\frac{\partial f}{\partial y}=-F_{r}^{e x t}(1+f) & \text { at } & y=0, \\
f \rightarrow 0 & \text { as } & y \rightarrow \infty .
\end{array}
$$

If it is assumed that $\hat{\boldsymbol{F}}^{\text {ext }}=-\hat{z}$, so that $F_{r}^{\text {ext }}=-\cos \theta$ and $F_{\theta}^{\text {ext }}=\sin \theta$, we recover the boundary-layer equation (5.8) presented in $\S 5.2$.

\section{Appendix D. Boundary-layer analysis of the pair-distribution function at high $P e_{b}$ in the absence of hydrodynamic interactions}

Here, we derive the expression (6.2) for the pair-distribution function, $g(s)$, at high $P e_{b}$ in the absence of hydrodynamic interactions. Without hydrodynamics, $g(s)$ satisfies

$$
\begin{aligned}
-P e_{b} \hat{\boldsymbol{F}}^{e x t} \cdot \nabla g & =\nabla^{2} g, \\
-P e_{b} \hat{\boldsymbol{s}} \cdot \hat{\boldsymbol{F}}^{e x t} g & =\frac{\partial g}{\partial s} \quad \text { at } \quad s=2, \\
g \rightarrow 1 & \text { as } s \rightarrow \infty .
\end{aligned}
$$

For $P e_{b} \gg 1$, advection dominates the microstructural deformation and, except near the probe, (D $1 a)$ reduces to $\hat{\boldsymbol{F}}^{\text {ext }} \cdot \nabla g=0$. This simply states that along a 'streamline' $g$ is constant, and the far-field boundary condition dictates the constant to be unity. However, this constant solution does not satisfy the no-flux boundary condition at contact and near the probe there exists a boundary layer in which Brownian diffusion balances advection. To focus on the boundary layer we introduce the stretched, or 
inner, coordinate $y=P e_{b}(s-2)$. The Smoluchowski equation now reads

$$
\begin{aligned}
P e_{b}^{2}\left(1-P e_{b}^{-2} y^{2}\right) \frac{\partial^{2} g}{\partial y^{2}} & +P e_{b}\left(1-P e_{b}^{-1} y\right) \frac{\partial g}{\partial y}+\frac{1-P e_{b}^{-1} y}{4 \sin \theta} \frac{\partial}{\partial \theta}\left(\sin \theta \frac{\partial g}{\partial \theta}\right) \\
& =-P e_{b}^{2} F_{r}^{e x t} \frac{\partial g}{\partial y}-\frac{P e_{b} F_{\theta}^{e x t}}{2}\left(1-\frac{P e_{b}^{-1} y}{2}\right) \frac{\partial g}{\partial \theta},
\end{aligned}
$$

the boundary conditions on $g$ are

$$
\begin{aligned}
-F_{r}^{e x t} g & =\frac{\partial g}{\partial y} \text { at } y=0, \\
g & \rightarrow 1 \text { as } y \rightarrow \infty .
\end{aligned}
$$

Inside the boundary layer we pose the expansion $g\left(y, \theta ; P e_{b}\right)=g_{1}(y, \theta) P e_{b}+g_{2}(y, \theta)+$ $O\left(P e_{b}^{-1}\right)$. Inserting the expansion into (D2) yields equations for $g_{1}$ and $g_{2}$. For $g_{1}$ we have

$$
\begin{gathered}
\frac{\partial^{2} g_{1}}{\partial y^{2}}+F_{r}^{e x t} \frac{\partial g_{1}}{\partial y}=0, \\
\frac{\partial g_{1}}{\partial y}+F_{r}^{e x t} g_{1}=0 \text { at } y=0, \\
g_{1} \rightarrow 0 \text { as } y \rightarrow \infty,
\end{gathered}
$$

which has the solution

$$
g_{1}(y, \theta)=A(\theta) \exp \left(-F_{r}^{e x t} y\right)
$$

where the angular function $A(\theta)$ will be found at the next order. This solution is only valid in the compressional region around the probe particle $\left(F_{r}^{e x t}>0\right)$, in which inward radial advection $\left(F_{r}^{e x t} \partial g_{1} / \partial y\right)$ balances Brownian diffusion $\left(\partial^{2} g_{1} / \partial y^{2}\right)$ leading to an $O\left(P e_{b}\right)$ build-up of pair density in the $O\left(\mathrm{Pe}_{b}^{-1}\right)$ thin boundary layer. In the extensional region, $F_{r}^{\text {ext }}<0$, force-free particles are advected away from the probe and there exists a wake in which $g \approx 0$.

The equation for $g_{2}$ is

$$
\begin{gathered}
\frac{\partial^{2} g_{2}}{\partial y^{2}}+F_{r}^{e x t} \frac{\partial g_{2}}{\partial y}=-\frac{\partial g_{1}}{\partial y}-\frac{F_{\theta}^{e x t}}{2} \frac{\partial g_{1}}{\partial \theta}, \\
\frac{\partial g_{2}}{\partial y}+F_{r}^{e x t} g_{2}=0 \quad \text { at } \quad y=0, \\
g_{2} \rightarrow 1 \text { as } y \rightarrow \infty .
\end{gathered}
$$

The far-field condition on $g_{2}$ ensures correct matching to the constant outer solution of unity. Some straightforward working gives

$$
g_{2}(y, \theta)=\left(\beta_{1}(\theta)+\beta_{2}(\theta) y+\beta_{3}(\theta) y^{2}\right) \exp \left(-F_{r}^{e x t} y\right)-\frac{\beta_{2}(\theta)}{F_{r}^{\text {ext }}},
$$

where

$$
\beta_{1}(\theta)=\frac{F_{\theta}}{2\left(F_{r}^{e x t}\right)^{2}} \frac{\mathrm{d} A(\theta)}{\mathrm{d} \theta}-\frac{A(\theta)}{F_{r}^{e x t}}\left(1+\frac{F_{\theta}}{2\left(F_{r}^{e x t}\right)^{2}} \frac{\mathrm{d} F_{r}^{e x t}}{\mathrm{~d} \theta}\right)-\frac{B(\theta)}{F_{r}^{e x t}},
$$




$$
\begin{aligned}
& \beta_{2}(\theta)=\frac{F_{\theta}^{e x t}}{2 F_{r}^{\text {ext }}} \frac{\mathrm{d} A(\theta)}{\mathrm{d} \theta}-A(\theta)\left(1+\frac{F_{\theta}}{2\left(F_{r}^{\text {ext }}\right)^{2}} \frac{\mathrm{d} F_{r}^{\text {ext }}}{\mathrm{d} \theta}\right), \\
& \beta_{3}(\theta)=-\frac{F_{\theta}^{\text {ext }}}{4 F_{\theta}^{\text {ext }}} \frac{\mathrm{d} F_{r}^{\text {ext }}}{\mathrm{d} \theta} A(\theta) .
\end{aligned}
$$

To determine the angular function $B(\theta)$, the perturbation expansion must be continued to the next order in $P e_{b}$; however, this is not important for our current purposes. The far-field boundary condition on $g_{2}$ is satisfied if $\beta_{2}(\theta)=-F_{r}^{e x t}$, which yields an equation for $A(\theta)$

$$
\frac{F_{\theta}^{e x t}}{2 F_{r}^{e x t}} \frac{\mathrm{d} A(\theta)}{\mathrm{d} \theta}-A(\theta)\left(1+\frac{F_{\theta}}{2\left(F_{r}^{e x t}\right)^{2}} \frac{\mathrm{d} F_{r}^{\text {ext }}}{\mathrm{d} \theta}\right)=-F_{r}^{\text {ext }},
$$

which has the solution $A(\theta)=F_{r}^{e x t}$. Thus, the pair-distribution function in the boundary layer is $g\left(y, \theta ; P e_{b}\right)=P e_{b} F_{r}^{e x t} e^{-F_{r}^{e x t} y}+O(1)$, which shows that there is an $O\left(P e_{b}\right)$ excess of force-free particles in the compressional region around the probe. From (3.15) we see that, at high $P e_{b}$, the intrinsic microviscosity is

$$
\eta_{i}=\frac{3}{4 \pi} \frac{2}{P e_{b}} \hat{\boldsymbol{F}}^{e x t} \cdot \oint_{s=2} g(s) \hat{\boldsymbol{s}} \mathrm{d} \Omega=\frac{3}{2 \pi} \int_{F_{r}^{e x t}>0} F_{r}^{\text {ext }} F_{r}^{\text {ext }} \mathrm{d} \Omega+O\left(P e_{b}^{-1}\right) .
$$

Taking $\hat{\boldsymbol{F}}^{\text {ext }}=-\hat{z}$ (so that $F_{r}^{e x t}=-\cos \theta$ ) gives $\eta_{i}^{P}=1+O\left(P e_{b}^{-1}\right.$ ) as $P e_{b} \rightarrow \infty$.

\section{Appendix E. Boundary-layer analysis of the pair-distribution function at high $P e_{b}$ for $\hat{b} \equiv 1$}

In this Appendix, we derive the scaling result $g(2) \sim P e_{b}^{0.799}$ used in $\S 6.2$ in discussing the contact value of the pair-distribution function at high $P e_{b}$ in the limit $\hat{b} \rightarrow 1$.

For large $P e_{b}$, the Smoluchowski equation (5.1) displays the familiar trait of a small parameter multiplying the highest-order derivative of a differential equation. Thus, we expect the solution about $P e_{b}^{-1} \equiv 0$ to be singular with an 'inner' region (or boundary layer) adjacent to the probe particle in which the effects of Brownian motion and advection are of comparable magnitude. Outside the boundary layer, where advection is dominant, Batchelor (1982) finds that the pair-distribution function is

$$
\ln g(s)=\int_{s}^{\infty}\left(\frac{2(G-H)}{z G}+\frac{1}{G} \frac{\mathrm{d} G}{\mathrm{~d} z}\right) \mathrm{d} z
$$

which is spherically symmetric and satisfies the boundary condition at infinity. However, in the limit $s \rightarrow 2$

$$
g(\xi) \sim g_{0} \xi^{H_{0} / G_{1}-1}\left(\ln \left(\xi^{-1}\right)\right)^{-H_{1} / G_{1}}
$$

where $\xi=s-2, g_{0}$ is a constant, and $H_{0}=0.402$ and $G_{1}=2$ are leading-order terms in the expansion of the hydrodynamic functions $H(s)$ and $G(s)$, respectively, about $s=2$. This expression is singular as $s \rightarrow 2$ and we must take into account the effects of Brownian diffusion to satisfy the inner boundary condition. Balancing terms in (5.1) suggests that diffusion balances advection when $s \sim O\left(P e_{b}^{-1}\right)$; defining an inner radial coordinate $y=P e_{b}(s-2)$, we obtain to leading order in $P e_{b}$ the boundary-layer equation

$$
\frac{\partial}{\partial y}\left(G_{1} y \frac{\partial g}{\partial y}\right)-G_{1} \cos \theta y \frac{\partial g}{\partial y}+\frac{H_{0}}{2} \sin \theta \frac{\partial g}{\partial \theta}-W_{0} \cos \theta g=0,
$$


where $W_{0}=G_{1}-H_{0}$. The first term in (E 3) represents radial diffusion of pair density with a linearly increasing diffusivity $G_{1} y$. The second and third terms denote the radial and angular advection of pair density, respectively, via the relative velocity field. Finally, the last term corresponds to a dipole source of pair density. The boundary conditions that a solution of (E 3) must satisfy are

$$
\begin{aligned}
g(y, \theta) & \rightarrow g_{0} P e_{b}^{W_{0} / G_{1}} y^{-W_{0} / G_{1}} \quad \text { as } \quad y \rightarrow \infty, \\
y \frac{\partial g}{\partial y}=0 \quad \text { at } \quad y=0 . &
\end{aligned}
$$

The right-hand side of (E $4 a$ ) is the inner limit of Batchelor's solution at infinite Péclet number (E 2), modulo a weak multiplicative correction of $O\left(\left(\ln \left(P e_{b} / y\right)\right)^{-H_{1} / G_{1}}\right)$ to which it is not possible to match at this order. To solve the boundary-layer problem, we propose the similarity solution $g(y, \theta)=h(\theta) p(\eta)$, where $\eta=y / Y(\theta)$ is the similarity variable. Substituting this ansatz into (E 3), we find that

$\eta \frac{\mathrm{d}^{2} p}{\mathrm{~d} \eta^{2}}+\left\{1-\left(\cos \theta Y+\frac{H_{0}}{2 G_{1}} \sin \theta \frac{\mathrm{d} Y}{\mathrm{~d} \theta}\right) \eta\right\} \frac{\mathrm{d} p}{\mathrm{~d} \eta}+\left(\frac{H_{0}}{2 G_{1}} \sin \theta Y \frac{\mathrm{d} \ln h}{\mathrm{~d} \theta}-\frac{W_{0}}{G_{1}} \cos \theta Y\right) p=0$,

subject to the boundary conditions

$$
\begin{aligned}
h(\theta) p(\eta) & \rightarrow g_{0} P e_{b}^{W_{0} / G_{1}} y^{-W_{0} / G_{1}} \quad \text { as } \quad \eta \rightarrow \infty, \\
\eta \frac{\partial g}{\partial \eta} & =0 \quad \text { at } \quad \eta=0 .
\end{aligned}
$$

For the similarity transformation to be successful, we require that

$$
\begin{array}{r}
\frac{H_{0}}{2 G_{1}} \sin \theta \frac{\mathrm{d} Y}{\mathrm{~d} \theta}+\cos \theta Y=\alpha, \\
\frac{H_{0}}{2 G_{1}} \sin \theta Y \frac{\mathrm{d} \ln h}{\mathrm{~d} \theta}-\frac{W_{0}}{G_{1}} \cos \theta Y=\beta,
\end{array}
$$

for constants $\alpha$ and $\beta$. Setting $\theta=\pi$ in the above equations, we find that $\alpha / \beta=$ $-G_{1} / W_{0}$. The equation for the similarity function $p$ then becomes

$$
\bar{\eta} \frac{\mathrm{d}^{2} p}{\mathrm{~d} \bar{\eta}^{2}}+(1-\bar{\eta}) \frac{\mathrm{d} p}{\mathrm{~d} \bar{\eta}}-\frac{W_{0}}{G_{1}} g=0,
$$

with $\bar{\eta}=\alpha \eta$. The solution to (E 8) is

$$
p(\eta)=c_{1} M\left(\frac{W_{0}}{G_{1}}, 1, \alpha \eta\right)+c_{2} U\left(\frac{W_{0}}{G_{1}}, 1, \alpha \eta\right),
$$

where $M(a, b, z)$ (the Kummer function) and $U(a, b, z)$ (the Tricomi function) are confluent hypergeometric functions. For a second argument of unity, the function $U$ is logarithmically singular at $\eta=0$ and hence is discarded. To satisfy the far-field condition (E $6 a$ ), we note that for $|z| \rightarrow \infty$ and $\operatorname{Re}(z)<0$ (which restricts $\alpha$ to be negative, Abramowitz \& Stegun 1972)

$$
M(a, b, z)=\frac{\Gamma(b)}{\Gamma(b-a)}(-z)^{-a}\left(1+O\left(|z|^{-1}\right)\right),
$$

where $\Gamma(z)$ is the gamma function. From (E $6 a)$, we find

$$
c_{1}=g_{0} \Gamma\left(\frac{H_{0}}{L_{1}}\right)\left(-\alpha P e_{b}\right)^{W_{0} / G_{1}} \frac{Y^{-W_{0} / G_{1}}}{h},
$$


which, for $c_{1}$ to indeed be a constant requires $h(\theta)=Y^{-W_{0} / G_{1}}$, compatible with (E 7a) and $(\mathrm{E} 7 b)$. Thus, we arrive at the boundary-layer solution for the pair-distribution function

$$
g(y, \theta)=g_{0} \Gamma\left(\frac{H_{0}}{G_{1}}\right)\left(\frac{-\alpha P e_{b}}{Y(\theta)}\right)^{W_{0} / G_{1}} M\left(\frac{W_{0}}{G_{1}}, 1, \frac{\alpha y}{Y(\theta)}\right) .
$$

Evidently, the function $Y(\theta)$ plays the role of the boundary-layer thickness and is found to be

$$
Y(\theta)=\frac{-2 \alpha G_{1}}{H_{0}}(\sin \theta)^{-2 G_{1} / H_{0}} \int_{\theta}^{\pi}(\sin \phi)^{2 G_{1} / H_{0}-1} \mathrm{~d} \phi .
$$

We may show that $-Y / \alpha=1$ at $\theta=\pi$ and that $Y>0$ for $0<\theta \leqslant \pi$ so that the boundary layer starts smoothly at $\theta=\pi$ and is well behaved for $0<\theta \leqslant \pi$. As $\theta \rightarrow 0$, the boundary-layer thickness diverges as $Y \sim \theta^{-2 G_{1} / H_{0}}$. This breakdown of the boundarylayer solution may be traced to the neglect of angular diffusion terms in (E 3), which are expected to be important near $\theta=0$ in describing the coalescence of the boundary layer into a wake behind the probe. Simple scaling arguments indicate that to retain the angular diffusion terms we must introduce the variable $x=P e_{b}^{1 / 2} \theta$ in addition to the radial boundary-layer coordinate $y=P e_{b}(s-2)$. This suggests that the coalescence region is of size $O\left(P e_{b}^{-3 / 2}\right)$ and hence 'smaller' than the $O\left(P e_{b}^{-1}\right)$ boundary layer.

Finally, we note that the contact $(y=0)$ value of the pair-distribution function is

$$
g(0, \theta)=g_{0} \Gamma\left(\frac{H_{0}}{G_{1}}\right)\left(\frac{-\alpha P e_{b}}{Y(\theta)}\right)^{W_{0} / G_{1}},
$$

from which we obtain the important scaling relation $g \sim P e_{b}^{0.799}$ in the boundary layer. This is in good agreement with the numerical scaling relation $g \sim P e_{b}^{0.773}$ determined from the finite-difference solution of the Smoluchowski equation at $\hat{b}=1.00001$ (cf. figure 14).

\section{REFERENCES}

Abramowitz, M. \& Stegun, I. 1972 Handbook of Mathematical Functions. Dover.

ACrivos, A \& TAYloR, T. D. 1965 Heat and mass transfer from single spheres in Stokes flow. Phys. Fluids 5, 387-394.

AlmoG, Y. \& BrenNER, H. 1997 Non-continuum anomalies in the apparent viscosity experienced by a test sphere moving through an otherwise quiescent suspension. Phys. Fluids 9, 16-22.

Barnes, H. A., Hutton, J. F. \& Walters, K. 1989 An Introduction to Rheology. Elsevier.

BATCHELOR, G. K. 1976 Brownian diffusion of particles with hydrodynamic interaction. J. Fluid Mech. 74, 1-29.

BAtChelor, G. K. 1982 Sedimentation in a dilute polydisperse system of interacting spheres. Part 1. General theory. J. Fluid Mech. 119, 379-408.

Batchelor, G. K. 1983 Diffusion in a dilute polydisperse system of interacting spheres. J. Fluid Mech. 131, 155-176.

Batchelor, G. K. \& Wen, C. S. 1982 Sedimentation in a dilute polydisperse system of interacting spheres. Part 2. Numerical results. J. Fluid Mech. 124, 495-528.

Bergenholtz, J., Brady, J. F. \& Vicic, M. 2002 The non-Newtonian rheology of dilute colloidal suspensions. J. Fluid Mech. 456, 239-275.

Brady, J. F. 1994 The long-time self-diffusivity in concentrated colloidal dispersions. J. Fluid Mech. 272, 109-133.

Brady, J. F. \& Morris, J. F. 1997 Microstructure of strongly sheared suspensions and its impact on rheology and diffusion. J. Fluid Mech. 348, 103-139. 
Crocker, J. C., Valentine, M. T., Weeks, E. R., Gisler, T., Kaplan, P. D., Yodh, A. G. \& Weitz, D. A. 2000 Two-point microrheology of inhomogeneous soft materials. Phys. Rev. Lett. 84, 888-891.

Davis, R. H. \& Hill, N. A. 1992 Hydrodynamic diffusion of a sphere sedimenting through a dilute suspension of neutrally buoyant spheres. J. Fluid Mech. 236, 513-533.

Foss, D. R. \& Brady, J. F. 2000 Structure, diffusion and rheology of Brownian suspensions by Stokesian Dynamics simulation. J. Fluid Mech. 407, 167-200.

Gittes, F., Schnurr, B., Olmsted, P. D., MacKintosh, F. C. \& Schimdt, C. F. 1997 Microscopic viscoelasticity: shear moduli of soft materials determined from thermal fluctuations. Phys. Rev. Lett. 79, 3286-3289.

Habdas, P., SchaAR, D., Levitt, A. C. \& Weeks, E. R. 2004 Forced motion of a probe particle near the colloidal glass transition. Europhys. Lett. 67, 477-483.

JeFFREY, D. J. \& ONISHI, Y. 1984 Calculation of the resistance and mobility functions of two unequal rigid spheres in low-Reynolds-number flow. J. Fluid Mech. 139, 261-290.

Kim, S. \& Karilla, S. J. 1991 Microhydrodynamics: Principles and Selected Applications. Butterworth-Heinemann.

Lionberger, R. A. 1998 Shear thinning of colloidal dispersions. J. Rheol. 42, 843-863.

MacKintosh, F. C. \& Schimdt, C. F. 1999 Microrheology. Curr. Opin. Colloid Interface Sci. 4, 300-307.

Mason, T. G., Ganesan, K., van Zanten, J. H., Wirtz, D. \& Kuo, S. C. 1997 Particle tracking microrheology of complex fluids. Phys. Rev. Lett. 79, 3282-3285.

Mason, T. G. \& Weitz, D. A. 1995 Optical measurements of frequency-dependent linear viscoelastic moduli of complex fluids. Phys. Rev. Lett. 74, 1250-1253.

Morris, J. F. \& KatYAL, B. 2002 Microstructure from simulated Brownian suspension flows at large shear rate. Phys. Fluids 14, 1920-1937.

NG, K. C. 1974 Hypernetted chain solutions for the classical one component plasma up to $\Gamma=7000$. J. Chem. Phys. 61, 2680-2689.

Press, W. H., Teukolsky, S. A., Vetterling, W. T. \& Flannery, B. P. 1992 Numerical Recipes in FORTRAN, 2nd edn. Cambridge University Press.

Rallison, J. M. \& Hinch, E. J. 1986 The effect of particle interactions on dynamic light scattering from a dilute suspension. J. Fluid Mech. 167, 131-168.

Russel, W. B., Saville, D. A. \& Schowalter, W. R. 1989 Colloidal Dispersions. Cambridge University Press.

Squires, T. M. \& Brady, J. F. 2005 A simple paradigm for active and nonlinear microrheology. Phys. Fluids 17, 073101.

Sutherland, W. 1905 A dynamical theory of diffusion for nonelectrolytes and the molecular mass of albumin. Phil. Mag. 6, 781-785.

VAN DyKe, M. 1975 Perturbation Methods in Fluid Mechanics. Parabolic Press.

Waigh, T. A. 2005 Microrheology of complex fluids. Rep. Prog. Phys. 68, 685-742. 